\title{
PERTURBATION ANALYSIS OF MATRICES OVER A QUATERNION DIVISION ALGEBRA*
}

\author{
SK. SAFIQUE AHMAD ${ }^{\dagger}$, ISTKHAR ALI ${ }^{\ddagger}$, AND IVAN SLAPNIČAR ${ }^{\S}$
}

\begin{abstract}
In this paper, we present the concept of perturbation bounds for the right eigenvalues of a quaternionic matrix. In particular, a Bauer-Fike-type theorem for the right eigenvalues of a diagonalizable quaternionic matrix is derived. In addition, perturbations of a quaternionic matrix are discussed via a block-diagonal decomposition and the Jordan canonical form of a quaternionic matrix. The location of the standard right eigenvalues of a quaternionic matrix and a sufficient condition for the stability of a perturbed quaternionic matrix are given. As an application, perturbation bounds for the zeros of quaternionic polynomials are derived. Finally, we give numerical examples to illustrate our results.
\end{abstract}

Key words. quaternionic matrices, left eigenvalues, right eigenvalues, quaternionic polynomials, Bauer-Fike theorem, quaternionic companion matrices, quaternionic matrix norms

AMS subject classifications. 15A18, 15A66

1. Introduction. The goal of this paper is the derivation of a Bauer-Fike-type theorem for the right eigenvalues and a perturbation analysis for quaternionic matrices, as well as a specification of the location of the right eigenvalues of a perturbed quaternionic matrix and perturbation bounds for the zeros of quaternionic polynomials. The Bauer-Fike theorem is a standard result in the perturbation theory for diagonalizable matrices over the complex field. The theorem states that if $A \in M_{n}(\mathbb{C})$ is a diagonalizable matrix, with $A=X D X^{-1}$, and $A+E$ is a perturbed matrix, then an upper bound for the distance between a point $\mu \in \Lambda(A+E)$ and the spectrum $\Lambda(A)$ is given by [4]

$$
\min _{\lambda \in \Lambda(A)}|\mu-\lambda| \leq \kappa(X)\|E\| .
$$

Here, $\kappa(X)=\|X\|\left\|X^{-1}\right\|$ is the condition number of the matrix $X$. Applications of the Bauer-Fike theorem over the complex field have been given in $[6,11,21,22,30]$. In general, a quaternionic matrix similarity is meaningless for the left eigenvalues. However, there are many results for quaternionic matrices on the similarity and the diagonalizability with respect to the right eigenvalues.

Many authors have extended various results from the complex field to the non-commutative skew field of the quaternions, for instance, the Jordan canonical form, the Schur decomposition, the singular-value decomposition, the diagonalization of quaternionic matrices, etc.; see $[9,17,27,28,33]$. On the other hand, Bauer-Fike-type results, a perturbation analysis of quaternionic matrices, and a perturbation analysis of the zeros of polynomials over a skew field have not yet been studied, even though the corresponding theory over the complex field is well known in the literature. The perturbation analysis over the skew field of quaternions is important in quantum physics, control theory, and mechanics; see, e.g., [1, 19, 25, 28].

Recently, some work on the location of zeros and on finding the zeros of quaternionic polynomials became popular in the literature. Many research papers dealing with the problem

* Received January 15, 2019. Accepted July 13, 2020. Published online on January 22, 2021. Recommended by Dario Bini. Research work of Istkhar Ali is funded by the CSIR, Govt. of India.

$\dagger$ Department of Mathematics, Indian Institute of Technology Indore, Khandwa Road, Simrol, Indore 453552, Madhya Pradesh, India (safique@itti.ac.in, safique@gmail.ac.in).

*Department of Mathematics \& Statistics, Integral University, Dasauli, Kursi Road, Lucknow (U.P.)-226026, India. (iaansari1986@gmail . com).

${ }^{\S}$ Faculty of Electrical Engineering, Mechanical Engineering and Naval Architecture, University of Split, Rudjera Boškovića 32, 21000 Split, Croatia (ivan. slapnicarefesb.hr). 
of finding the zeros of quaternionic polynomials are available; see, e.g., [7, 12, 15, 16, 23, $24,26,29]$. The focus is put on finding bounds and the location of the zeros of quaternionic polynomials, which both are important research topics; see, e.g., [3, 18, 24].

In this paper, we extend the Bauer-Fike theorem over the complex field to the noncommutative skew-field of quaternions. In particular, a Bauer-Fike-type theorem for the right eigenvalues of a quaternionic diagonalizable matrix is derived. The results on the perturbation theory of quaternionic matrices are presented utilizing the Jordan canonical form and the block-diagonal decomposition form of quaternionic matrices. Also, the localization theorems for the right eigenvalues of quaternionic matrices and a sufficient condition for the stability of a perturbed quaternionic matrix are derived. An application of the aforementioned results about perturbations of quaternionic matrices is the derivation of perturbation bounds for the zeros of quaternionic polynomials of the form

$$
\begin{aligned}
& p_{l}(z)=q_{m} z^{m}+q_{m-1} z^{m-1}+\cdots+q_{1} z+q_{0}, \\
& p_{r}(z)=z^{m} q_{m}+z^{m-1} q_{m-1}+\cdots+z q_{1}+q_{0},
\end{aligned}
$$

where $q_{j}, z \in \mathbb{H},(0 \leq j \leq m)$. The polynomials (1.2) and (1.3) are called "simple", and "monic" if $q_{m}=1$.

The paper is organized as follows. In Section 2, the notation and a few results from $[9,20$, 33] are given. A Bauer-Fike-type theorem and perturbation theorems for the right eigenvalues of quaternionic matrices are derived in Section 3. The localization theorems for the right eigenvalues and a sufficient condition for the stability of a perturbed quaternionic matrix are also given in Section 3. Section 4 is devoted to the perturbation analysis of quaternionic polynomials. Finally, in Section 5, examples are given to illustrate our results.

2. Notation and preliminaries. Throughout this paper, the following notation and terminology are adopted. $\mathbb{R}$ and $\mathbb{C}$ denote the fields of real and complex numbers, respectively. The set of real quaternions is defined as

$$
\mathbb{H}=\left\{q=a_{0}+a_{1} \mathbf{i}+a_{2} \mathbf{j}+a_{3} \mathbf{k}: a_{0}, a_{1}, a_{2}, a_{3} \in \mathbb{R}\right\},
$$

where $\mathbf{i}^{2}=\mathbf{j}^{2}=\mathbf{k}^{2}=\mathbf{i j k}=-1$. For $q \in \mathbb{H}$, the conjugate and the modulus of $q$ are defined by $\bar{q}=a_{0}-a_{1} \mathbf{i}-a_{2} \mathbf{j}-a_{3} \mathbf{k}$ and $|q|:=\sqrt{a_{0}^{2}+a_{1}^{2}+a_{2}^{2}+a_{3}^{2}}$, respectively. $\Re(a)$ and $\Im(a)$ denote the real and imaginary parts of $a \in \mathbb{C}$, respectively. The real part of a quaternion $q=a_{0}+a_{1} \mathbf{i}+a_{2} \mathbf{j}+a_{3} \mathbf{k}$ is defined as $\operatorname{real}(q)=a_{0}$. Let $\mathbb{H}^{n}$ be the collection of all $n$-column vectors with entries in $\mathbb{H}$. For $x \in \mathbb{H}^{n}$, the transpose of $x$ is $x^{T}$. If $x=\left[x_{1}, \ldots, x_{n}\right]^{T}$, then the conjugate of $x$ is defined as $\bar{x}=\left[\overline{x_{1}}, \ldots, \overline{x_{n}}\right]^{T}$, and the conjugate transpose of $x$ is defined as $x^{H}=\left[\overline{x_{1}}, \ldots, \overline{x_{n}}\right]$. For $x, y \in \mathbb{H}^{n}$, let $\langle x, y\rangle:=y^{H} x$ be the inner product and $\|x\|_{2}=\sqrt{\langle x, x\rangle}$ be the norm in $\mathbb{H}^{n}$. The sets of $m \times n$ real, complex, and quaternionic matrices are denoted by $M_{m \times n}(\mathbb{R}), M_{m \times n}(\mathbb{C})$, and $M_{m \times n}(\mathbb{H})$, respectively. These sets are simply denoted by $M_{n}(\mathcal{K}), \mathcal{K} \in\{\mathbb{R}, \mathbb{C}, \mathbb{H}\}$, when $m=n$. $I_{n}$ denotes the $n \times n$ identity matrix. For $A=\left(a_{i j}\right) \in M_{m \times n}(\mathcal{K})$, the conjugate, transpose, and conjugate transpose of $A$ are defined as $\bar{A}=\left(\overline{a_{i j}}\right), A^{T}=\left(a_{j i}\right) \in M_{n \times m}(\mathcal{K})$, and $A^{H}=(\bar{A})^{T} \in M_{n \times m}(\mathcal{K})$, respectively. For $A \in M_{n}(\mathcal{K})$, the Frobenius norm and the spectral norm of $A$ are defined as

$$
\|A\|_{F}=\left[\operatorname{trace}\left(A^{H} A\right)\right]^{1 / 2} \quad \text { and } \quad\|A\|_{2}=\max \left\{\|A x\|_{2}: x \in \mathbb{H}^{n},\|x\|_{2}:=1\right\},
$$

respectively. A matrix $A \in M_{n}(\mathbb{H})$ is said to be Hermitian if $A^{H}=A$, normal if $A^{H} A=A A^{H}$, and invertible (nonsingular) if $A B=B A=I$ for some $B \in M_{n}(\mathbb{H})$, where $I$ is the identity matrix. The closed upper half plane in the complex plane is denoted by $\mathbb{C}^{+}=\{\alpha+\beta i: \alpha, \beta \in \mathbb{R}, \beta \geq 0\}$ and the nonnegative reals by $\mathbb{R}^{+}=\{\alpha: \alpha \in \mathbb{R}, \alpha \geq 0\}$. 
Let $A \in M_{n}(\mathbb{H})$ be an invertible matrix. Then the condition number of the matrix $A$ is defined as $\kappa(A)=\|A\|_{2}\left\|A^{-1}\right\|_{2}$. The set of zeros of a quaternionic polynomial $p(z)$ is denoted by $Z_{\mathbb{H}}(p(z))$. The set of complex zeros of a quaternionic polynomial $p(z)$ is denoted by $Z_{\mathbb{C}}(p(z))$. A Jordan block of size $m$ associated with $\lambda \in \mathbb{H}$ is defined as

$$
J_{m}(\lambda)=\left[\begin{array}{ccccc}
\lambda & 1 & 0 & \ldots & 0 \\
0 & \lambda & 1 & \ldots & 0 \\
\vdots & \vdots & \ddots & \ddots & 0 \\
\vdots & \vdots & & \lambda & 1 \\
0 & 0 & \ldots & 0 & \lambda
\end{array}\right] \in M_{m}(\mathbb{H}), \quad \lambda \in \mathbb{H}
$$

For $x \in \mathbb{H}^{n}$ and $A \in M_{n}(\mathbb{H})$, we define $\operatorname{real}(x)=\frac{1}{2}(x+\bar{x})$ and $\operatorname{real}(A)=\frac{1}{2}(A+\bar{A})$. Any quaternionic vector $x=y_{1}+y_{2} \mathbf{i}+y_{3} \mathbf{j}+y_{4} \mathbf{k} \in \mathbb{H}^{n}$, where $y_{k} \in \mathbb{R}^{n}$, for $k=1,2,3,4$, can be uniquely expressed as

$$
x=\left(y_{1}+y_{2} \mathbf{i}\right)+\left(y_{3}+y_{4} \mathbf{i}\right) \mathbf{j} \equiv x_{1}+x_{2} \mathbf{j}, \quad x_{1}, x_{2} \in \mathbb{C}^{n} .
$$

Define a function $\psi: \mathbb{H}^{n} \rightarrow \mathbb{C}^{2 n}$ by

$$
\psi_{x}=\left[\begin{array}{c}
x_{1} \\
-\overline{x_{2}}
\end{array}\right]
$$

The vector $\psi_{x}$ is called the complex adjoint vector of $x$. The function $\psi$ is an injective linear transformation from $\mathbb{H}^{n}$ to $\mathbb{C}^{2 n}$. The formulas for $x_{1}$ and $x_{2}$ are

$$
x_{1}=\operatorname{real}(x)-\operatorname{real}(x \mathbf{i}) \mathbf{i}, \quad x_{2}=-\operatorname{real}(x \mathbf{j})-\operatorname{real}(x \mathbf{k}) \mathbf{i} .
$$

Similarly, any quaternionic matrix $A=B_{1}+B_{2} \mathbf{i}+B_{3} \mathbf{j}+B_{4} \mathbf{k} \in M_{n}(\mathbb{H})$, where $B_{k} \in M_{n}(\mathbb{R})$, for $k=1,2,3,4$, can be uniquely expressed as

$$
A=\left(B_{1}+B_{2} \mathbf{i}\right)+\left(B_{3}+B_{4} \mathbf{i}\right) \mathbf{j} \equiv A_{1}+A_{2} \mathbf{j}, \quad A_{1}, A_{2} \in M_{n}(\mathbb{C}) .
$$

Define a function $\Psi: M_{n}(\mathbb{H}) \rightarrow M_{2 n}(\mathbb{C})$ by

$$
\Psi_{A}=\left[\begin{array}{cc}
\frac{A_{1}}{-A_{2}} & \frac{A_{2}}{A_{1}}
\end{array}\right]
$$

The matrix $\Psi_{A}$ is called the complex adjoint matrix of $A$. The function $\Psi$ is an injective $H$-homomorphism. The formulas for $A_{1}$ and $A_{2}$ are

$$
A_{1}=\operatorname{real}(A)-\operatorname{real}(A \mathbf{i}) \mathbf{i}, \quad A_{2}=-\operatorname{real}(A \mathbf{j})-\operatorname{real}(A \mathbf{k}) \mathbf{i} .
$$

Due to the non-commutativity of quaternions, there are two type of eigenvalues, namely the right and left eigenvalues, defined as follows:

DEFINITION 2.1. Let $A \in M_{n}(\mathbb{H})$. Then the left, the right, and the standard right eigenvalues, respectively, are defined by

$$
\begin{aligned}
& \Lambda_{l}(A)=\left\{\lambda \in \mathbb{H}: A x=\lambda x \text { for some nonzero } x \in \mathbb{H}^{n}\right\} \\
& \Lambda_{r}(A)=\left\{\lambda \in \mathbb{H}: A x=x \lambda \text { for some nonzero } x \in \mathbb{H}^{n}\right\}, \quad \text { and } \\
& \Lambda_{s}(A)=\left\{\lambda \in \mathbb{C}: A x=x \lambda \text { for some nonzero } x \in \mathbb{H}^{n}, \Im(\lambda) \geq 0\right\}
\end{aligned}
$$


Let $p, q \in \mathbb{H}$. Then $p$ and $q$ are said to be similar, denoted by $p \sim q$, if

$$
p \sim q \Leftrightarrow \exists 0 \neq r \in \mathbb{H} \text { such that } p=r^{-1} q r .
$$

The set

$$
[p]=\left\{u \in \mathbb{H}: u=\rho^{-1} p \rho \text { for all } 0 \neq \rho \in \mathbb{H}\right\}
$$

is called the equivalence class of $p \in \mathbb{H}$. It is well known [33, Theorem 2.2] that

$$
p \sim q \Leftrightarrow \operatorname{real}(p)=\operatorname{real}(q) \text { and }|p|=|q| .
$$

From (2.1), (2.2), and (2.3), $[p]$ can be written as

$$
[p]=\{x \in \mathbb{H}: \operatorname{real}(x)=\operatorname{real}(p),|x|=|p|\} .
$$

From (2.4), we have $\bar{p} \in[p]$.

DEFINITION 2.2. Let $A \in M_{n}(\mathbb{H})$. Then the quaternionic continuous-time system

$$
\frac{d}{d t} w(t)=A w(t)
$$

is stable if and only if $\Lambda_{r}(A) \subset \mathbb{H}^{-}=\{q \in \mathbb{H}: \operatorname{real}(q)<0\}$.

DEFINITION 2.3. Let $A \in M_{n}(\mathbb{H})$. Then the matrix $A$ is said to be nilpotent if there exists a positive integer $t$ such that $A^{t}=0$, where 0 is the zero matrix.

DEFINITION 2.4. Let $A \in M_{n}(\mathbb{H})$. Then $A$ is said to be a central closed matrix if there exists an invertible matrix $T$ such that $T^{-1} A T=\operatorname{diag}\left(\lambda_{1}, \lambda_{2}, \ldots, \lambda_{n}\right)$, where $\lambda_{i} \in \mathbb{R}$, $(1 \leq i \leq n)$.

Every quaternionic matrix $A \in M_{n}(\mathbb{H})$ has exactly $n$ right eigenvalues which are complex numbers with nonnegative imaginary parts. Those right eigenvalues are said to be the standard right eigenvalues of $A$. Moreover, if $A$ is in triangular form, then every diagonal entry is a right eigenvalue of $A$. Consequently, the $\lambda_{i}$ 's are standard right eigenvalues.

We now present some basic and known facts for a matrix $A \in M_{n}(\mathbb{H})$ and its complex adjoint matrix $\Psi_{A}$ for a further development of our theory.

THEOREM 2.5 ([33, Theorem 4.2]). Let $A, B \in M_{n}(\mathbb{H})$, and $\alpha \in \mathbb{R}$. Then

(a) $\Psi_{I_{n}}=I_{2 n}$.

(b) $\Psi_{A B}=\Psi_{A} \Psi_{B}$.

(c) $\Psi_{\alpha A}=\alpha \Psi_{A}$.

(d) $\Psi_{A+B}=\Psi_{A}+\Psi_{B}$.

(e) $\Psi_{A^{H}}=\left(\Psi_{A}\right)^{H}$.

(f) $\Psi_{A^{-1}}=\left(\Psi_{A}\right)^{-1}$ if $A^{-1}$ exists.

(g) $\Psi_{A}$ is unitary, Hermitian, diagonalizable, invertible, or normal if and only if $A$ is unitary, Hermitian, diagonalizable, invertible, or normal, respectively.

Lemma 2.6 ([20, Theorem 4.1]). Let $A \in M_{n}(\mathbb{H})$. Then $\Psi_{A} \in \mathbb{C}^{2 n}$ and

$$
\max _{\|x\|_{2} \neq 0} \frac{\|A x\|_{2}}{\|x\|_{2}}=\max _{\|y\|_{2} \neq 0} \frac{\left\|\Psi_{A} y\right\|_{2}}{\|y\|_{2}} .
$$

Next, we give a relation between the spectral norm and the Frobenius norm of a quaternionic matrix.

Lemma 2.7 ([2, Lemma 3.5]). Let $A \in M_{n}(\mathbb{H})$. Then $\|A\|_{2} \leq\|A\|_{F}$. 
In general, the trace of a quaternionic matrix is not equal to the sum of the standard right eigenvalues of that quaternionic matrix. For example, consider a quaternionic matrix

$$
A=\left[\begin{array}{ll}
0 & \mathbf{i} \\
\mathbf{j} & 0
\end{array}\right] \text {. }
$$

The set of right eigenvalues of $A$ is $\Lambda_{r}(A)=\left[\frac{\sqrt{2}}{2}(-1+\mathbf{i})\right] \cup\left[\frac{\sqrt{2}}{2}(1+\mathbf{i})\right]$. Clearly, the standard right eigenvalues of $A$ are $\lambda_{1}=\frac{\sqrt{2}}{2}(-1+\mathbf{i})$ and $\lambda_{2}=\frac{\sqrt{2}}{2}(1+\mathbf{i})$. Hence, $\operatorname{trace}(A)=0 \neq \sqrt{2} \mathbf{i}=\lambda_{1}+\lambda_{2}$. However, the trace of a quaternionic Hermitian matrix is equal to the sum of the standard right eigenvalues of that quaternionic Hermitian matrix. Thus, by the definition of the Frobenius norm of a quaternionic matrix $A \in M_{n}(\mathbb{H})$, we have

$$
\|A\|_{F}^{2}=\operatorname{trace}\left(A^{H} A\right)=\sum_{j=1}^{n} \lambda_{j}\left(A^{H} A\right) \leq n \lambda_{\max }\left(A^{H} A\right)=n\|A\|_{2}^{2},
$$

so that

$$
\|A\|_{F} \leq \sqrt{n}\|A\|_{2} .
$$

More interesting results like the Jordan canonical form, the singular-value decomposition, and the Schur decomposition of a quaternionic matrix may be found in $[9,27,31,32,33]$.

Proposition 2.8 ([27, Proposition 2.7]). Let $A \in M_{n}(\mathbb{H})$. Then there exists an invertible matrix $Y \in M_{n}(\mathbb{H})$ such that

$$
Y^{-1} A Y=\operatorname{diag}\left(J_{m_{1}}\left(\lambda_{1}\right), J_{m_{2}}\left(\lambda_{2}\right), \ldots, J_{m_{k}}\left(\lambda_{k}\right)\right),
$$

where $\lambda_{i} \in \mathbb{H}, \lambda_{i} \in \Lambda_{s}(A)(1 \leq i \leq k)$, and $J_{m_{i}}\left(\lambda_{i}\right)$ are $m_{i} \times m_{i}$ Jordan blocks with the right eigenvalues $\lambda_{i}$, respectively. Moreover, the right-hand side of (2.5) is uniquely determined by A up to a permutation of diagonal blocks and up to a replacement of each $\lambda_{j}$ with any similar quaternion $\mu_{j}$.

Lemma 2.9 ([33, Theorem 7.2]). Let $A \in M_{m \times n}(\mathbb{H})$ be of rank $r$. Then there exist two unitary matrices, $U \in M_{m}(\mathbb{H})$ and $V \in M_{n}(\mathbb{H})$, such that

$$
U^{H} A V=\left[\begin{array}{cc}
\Sigma_{r} & 0 \\
0 & 0
\end{array}\right]
$$

where $\Sigma_{r}=\operatorname{diag}\left(\sigma_{1}, \ldots, \sigma_{r}\right)$ and $\sigma_{i} \in \mathbb{R}, \sigma_{i}>0$, are the singular values of $A$. A also has $\min \{m, n\}-r$ singular values that are zero.

THEOREM 2.10 ([9, Lemma 3.2]). Let $A \in M_{n}(\mathbb{H})$. Then there exist matrices $T, V \in$ $M_{n}(\mathbb{H})$ such that

(a) $V^{H} A V=T$, where $V$ is a unitary matrix and $T$ is an upper triangular matrix,

(b) every diagonal entry of $T$ is contained in the closed upper complex half plane $\mathbb{C}^{+}$.

Theorem 2.11 ([9, Theorem 3.3]). Let $A \in M_{n}(\mathbb{H})$ be normal. Then there exist a unitary matrix $V \in M_{n}(\mathbb{H})$ and a diagonal matrix $D \in M_{n}(\mathbb{H})$ such that

(a) $V^{H} A V=D$,

(b) every diagonal entry of $D$ is contained in the closed upper complex half plane $\mathbb{C}^{+}$.

3. Perturbation analysis of quaternionic matrices. Let $A=\left(a_{i j}\right) \in M_{n}(\mathbb{H})$ be partitioned into $k \times k$ blocks,

$$
A=\left(A_{i j}\right)=\left[\begin{array}{cccc}
A_{11} & A_{12} & \ldots & A_{1 k} \\
A_{21} & A_{22} & \ldots & A_{2 k} \\
\vdots & \vdots & \ddots & \vdots \\
A_{k 1} & A_{k 2} & \ldots & A_{k k}
\end{array}\right]_{n \times n}
$$


where $A_{i, j} \in M_{n_{i}, n_{j}}(\mathbb{H})$ is the $(i, j)$-block of $A$ such that $n_{1}+\cdots+n_{k}=n$. We now derive some lemmas needed for the development of our theory.

Lemma 3.1. Let $a, b \in \mathbb{C}^{+}$. Then $|a-b| \leq|\bar{a}-b|$.

Proof. Suppose that $a=x+\mathbf{i} y$ and $b=p+\mathbf{i} q$, where $x, p \in \mathbb{R}$ and $y, q \in \mathbb{R}^{+}$. We have

$$
\begin{aligned}
& |a-b|=|x+\mathbf{i} y-(p+\mathbf{i} q)|=|(x-p)+\mathbf{i}(y-q)|, \\
& |\bar{a}-b|=|x-\mathbf{i} y-(p+\mathbf{i} q)|=|(x-p)+\mathbf{i}(y+q)| .
\end{aligned}
$$

It is known that if $y, q \in \mathbb{R}^{+}$, then $y+q \geq y-q$. Hence, from (3.1) and (3.2), we have $|a-b| \leq|\bar{a}-b|$.

LEMMA 3.2. Consider $\alpha_{k}, \beta_{k}, \gamma_{k} \in \mathbb{R}$, where $k=1,2$, and $\alpha_{1}^{2}+\beta_{1}^{2}+\gamma_{1}^{2}=1$ and $\alpha_{2}^{2}+\beta_{2}^{2}+\gamma_{2}^{2}=1$. Then, $\alpha_{1} \alpha_{2}+\beta_{1} \beta_{2}+\gamma_{1} \gamma_{2} \leq 1$.

Proof. We have

$$
\left(\alpha_{1}-\alpha_{2}\right)^{2}+\left(\beta_{1}-\beta_{2}\right)^{2}+\left(\gamma_{1}-\gamma_{2}\right)^{2}=2-2\left(\alpha_{1} \alpha_{2}+\beta_{1} \beta_{2}+\gamma_{1} \gamma_{2}\right) \geq 0,
$$

that is, $\alpha_{1} \alpha_{2}+\beta_{1} \beta_{2}+\gamma_{1} \gamma_{2} \leq 1$.

Lemma 3.3. Let $\lambda, \mu \in \mathbb{C}^{+}$, and let $\rho, \eta \in \mathbb{H} \backslash\{0\}$. Then $|\lambda-\mu| \leq\left|\rho^{-1} \lambda \rho-\eta^{-1} \mu \eta\right|$.

Proof. Consider

$$
\lambda=\lambda^{\prime}+\lambda^{\prime \prime} \mathbf{i}, \quad \mu=\mu^{\prime}+\mu^{\prime \prime} \mathbf{i}, \quad \rho^{-1} \mathbf{i} \rho=\alpha_{1} \mathbf{i}+\beta_{1} \mathbf{j}+\gamma_{\mathbf{1}} \mathbf{k}, \quad \eta^{-1} \mathbf{i} \eta=\alpha_{2} \mathbf{i}+\beta_{2} \mathbf{j}+\gamma_{\mathbf{2}} \mathbf{k},
$$

where $\alpha_{k}, \beta_{k}, \gamma_{k} \in \mathbb{R}$, for $k=1,2$, with $\alpha_{1}^{2}+\beta_{1}^{2}+\gamma_{1}^{2}=\alpha_{2}^{2}+\beta_{2}^{2}+\gamma_{2}^{2}=1$. Then

$$
P=|\lambda-\mu|^{2}=\left(\lambda^{\prime}-\mu^{\prime}\right)^{2}+\left(\lambda^{\prime \prime}\right)^{2}+\left(\mu^{\prime \prime}\right)^{2}-2 \lambda^{\prime \prime} \mu^{\prime \prime} .
$$

Also,

$$
\begin{aligned}
Q= & \left|\rho^{-1} \lambda \rho-\eta^{-1} \mu \eta\right|^{2} \\
= & \left(\lambda^{\prime}-\mu^{\prime}\right)^{2}+\left(\lambda^{\prime \prime} \alpha_{1}-\mu^{\prime \prime} \alpha_{2}\right)^{2}+\left(\lambda^{\prime \prime} \beta_{1}-\mu^{\prime \prime} \beta_{2}\right)^{2}+\left(\lambda^{\prime \prime} \gamma_{1}-\mu^{\prime \prime} \gamma_{2}\right)^{2} \\
= & \left(\lambda^{\prime}-\mu^{\prime}\right)^{2}+\left(\lambda^{\prime \prime}\right)^{2}\left[\alpha_{1}^{2}+\beta_{1}^{2}+\gamma_{1}^{2}\right]+\left(\mu^{\prime \prime}\right)^{2}\left[\alpha_{2}^{2}+\beta_{2}^{2}+\gamma_{2}^{2}\right] \\
& \quad-2 \lambda^{\prime \prime} \mu^{\prime \prime}\left[\alpha_{1} \alpha_{2}+\beta_{1} \beta_{2}+\gamma_{1} \gamma_{2}\right] .
\end{aligned}
$$

From Lemma 3.2, we have

$$
\alpha_{1} \alpha_{2}+\beta_{1} \beta_{2}+\gamma_{1} \gamma_{2}<1
$$

Thus $P \leq Q$, that is,

$$
|\lambda-\mu| \leq\left|\rho^{-1} \lambda \rho-\eta^{-1} \mu \eta\right|
$$

LEMMA 3.4. Let $A \in M_{n}(\mathbb{H})$ such that $A^{t}=0_{n}$ for some positive integer $t$, where $0_{n}$ is the $n \times n$ zero matrix. Then $\Psi_{A}^{t}=0_{2 n}$, where $0_{2 n}$ is the $2 n \times 2 n$ zero matrix.

Proof. Consider $A \in M_{n}(\mathbb{H})$ such that $A^{t}=0_{n}$ for some positive integer $t$. Then by taking the complex adjoint matrix of $A^{t}=0_{n}$ and by applying Theorem 2.5, we have $\Psi_{A^{t}}=\Psi_{0_{n}} \Rightarrow \Psi_{A}^{t}=0_{2 n}$. 
LEMMA 3.5. Let $T \in M_{n}(\mathbb{H})$ be partitioned as follows:

$$
T={ }_{q}^{p}\left[\begin{array}{cc}
p & q \\
T_{11} & T_{12} \\
0 & T_{22}
\end{array}\right] .
$$

Define the linear transformation $\phi: M_{p \times q}(\mathbb{H}) \rightarrow M_{p \times q}(\mathbb{H})$ by $\phi(X)=T_{11} X-X T_{22}$, where $X \in M_{p \times q}(\mathbb{H})$. Then $\phi$ is invertible if and only if $\Lambda_{r}\left(T_{11}\right) \cap \Lambda_{r}\left(T_{22}\right)=\emptyset$. If $\phi$ is invertible and $Y \in M_{n}(\mathbb{H})$ is defined by

$$
Y=\left[\begin{array}{cc}
I_{p} & Z \\
0 & I_{q}
\end{array}\right], \quad \phi(Z)=-T_{12}
$$

then $Y^{-1} T Y=\operatorname{diag}\left(T_{11}, T_{22}\right)$.

Proof. Let $X \in M_{p \times q}(\mathbb{H})$. Then from Lemma 2.9, we have

$$
U^{H} X V={ }_{p-r}^{r}\left[\begin{array}{cc}
\Sigma_{r} & q-r \\
0 & 0
\end{array}\right],
$$

where $\Sigma_{r}=\operatorname{diag}\left(\sigma_{i}\right)$ and $r=\operatorname{rank}(X)$. Assume $\phi(X)=0$ for $X \neq 0$. Substituting (3.3) into the quaternionic matrix equation $T_{11} X=X T_{22}$ yields

$$
\left[\begin{array}{ll}
A_{11} & A_{12} \\
A_{21} & A_{22}
\end{array}\right]\left[\begin{array}{cc}
\Sigma_{r} & 0 \\
0 & 0
\end{array}\right]=\left[\begin{array}{cc}
\Sigma_{r} & 0 \\
0 & 0
\end{array}\right]\left[\begin{array}{ll}
B_{11} & B_{12} \\
B_{21} & B_{22}
\end{array}\right]
$$

where $U^{H} T_{11} U=\left(A_{i j}\right)$ and $V^{H} T_{22} V=\left(B_{i j}\right)$. By comparing blocks we see that $A_{21}=0$, $B_{12}=0$, and $\Lambda_{r}\left(A_{11}\right)=\Lambda_{r}\left(B_{11}\right)$. Consequently,

$$
\emptyset \neq \Lambda_{r}\left(A_{11}\right)=\Lambda_{r}\left(B_{11}\right) \subseteq \Lambda_{r}\left(T_{11}\right) \cap \Lambda_{r}\left(T_{22}\right) .
$$

On the other hand, if $\lambda \in \Lambda_{r}\left(T_{11}\right) \cap \Lambda_{r}\left(T_{22}\right)$, then there exist nonzero vectors $x, y \in \mathbb{H}^{n}$ such that

$$
T_{11} x=x \lambda, \quad T_{22}^{H} y=y \lambda^{H} .
$$

Thus, $y^{H} T_{22}=\lambda y^{H}$, and hence $\phi\left(x y^{H}\right)=0$. Finally, if $\phi$ is invertible, then the quaternionic matrix $Z$ exists and

$$
\begin{aligned}
Y^{-1} T Y & =\left[\begin{array}{cc}
I & -Z \\
0 & I
\end{array}\right]\left[\begin{array}{cc}
T_{11} & T_{12} \\
0 & T_{22}
\end{array}\right]\left[\begin{array}{cc}
I & Z \\
0 & I
\end{array}\right] \\
& =\left[\begin{array}{cc}
T_{11} & T_{11} Z-Z T_{22}+T_{12} \\
0 & T_{22}
\end{array}\right]=\left[\begin{array}{cc}
T_{11} & 0 \\
0 & T_{22}
\end{array}\right] .
\end{aligned}
$$

We now establish a block diagonal decomposition of a quaternionic matrix, which reads as follows.

THEOREM 3.6 (Block diagonal decomposition). Suppose that

$$
U^{H} A U=T=\left[\begin{array}{cccc}
T_{11} & T_{12} & \ldots & T_{1 q} \\
0 & T_{22} & \ldots & T_{2 q} \\
\vdots & \vdots & \ddots & \vdots \\
0 & 0 & \ldots & T_{q q}
\end{array}\right]
$$

is a Schur decomposition of $A \in M_{n}(\mathbb{H})$, and assume that the diagonal blocks $T_{i i}$ are square. If $\Lambda_{r}\left(T_{i i}\right) \cap \Lambda_{r}\left(T_{j j}\right)=\emptyset$ whenever $i \neq j$, then there exists an invertible matrix $Y \in M_{n}(\mathbb{H})$ such that

$$
(U Y)^{-1} A(U Y)=\operatorname{diag}\left(T_{11}, \ldots, T_{q q}\right) .
$$

Proof. The proof is immediate by applying Lemma 3.5 and using induction. 
3.1. A Bauer-Fike-type theorem for the right eigenvalues of diagonalizable quaternionic matrices. In general, quaternionic matrix similarity is meaningless for the left eigenvalues of a quaternionic matrix. On the other hand, quaternionic matrix similarity exists for right eigenvalues. In addition, the Schur triangularization theorem for the right eigenvalues of a quaternionic matrix has been proved in [9]. In this section, we give an extension of the Bauer-Fike theorem (1.1). An extension of this theorem is not straightforward for the right eigenvalues of a quaternionic diagonalizable matrix. For example, if $\mu$ is a right eigenvalue of the perturbed quaternionic matrix $(A+\Delta A) \in M_{n}(\mathbb{H})$, then there exists a vector $x \in \mathbb{H}^{n}$ such that $(A+\Delta A) x=x \mu \Longrightarrow x \mu-A x=\Delta A x$. However, in general, we cannot write $(\mu I-A) x=\Delta A x$ or $x(\mu I-A)=\Delta A x$ due to the non-commutativity of the quaternions. Fortunately, we can extend the theorem by applying the complex adjoint matrix of a quaternionic matrix and Lemma 3.1, Lemma 2.6, and Lemma 3.3. We now derive the Bauer-Fike-type theorem for a diagonalizable quaternionic matrix.

THEOREM 3.7. Let $A \in M_{n}(\mathbb{H})$ be a diagonalizable matrix, that is, $A=Y \Lambda Y^{-1}$, where $Y \in M_{n}(\mathbb{H})$ is invertible and $\Lambda=\operatorname{diag}\left(\lambda_{1}, \ldots, \lambda_{n}\right)$ with $\lambda_{i}$ being the standard right eigenvalues of $A$. If $\mu$ is a standard right eigenvalue of $A+\Delta A$, then

$$
\operatorname{dist}\left(\mu, \Lambda_{s}(A)\right)=\min _{\lambda_{i} \in \Lambda_{s}(A)}\left\{\left|\lambda_{i}-\mu\right|\right\} \leq \kappa(Y)\|\Delta A\|_{2} .
$$

Moreover, we have

$$
\operatorname{dist}\left(\xi, \Lambda_{r}(A)\right)=\inf _{\eta_{j} \in \Lambda_{r}(A)}\left\{\left|\eta_{j}-\xi\right|\right\} \leq \kappa(Y)\|\Delta A\|_{2},
$$

where $\xi \in \Lambda_{r}(A+\Delta A)$ and $\kappa(\cdot)$ is the condition number with respect to the matrix 2-norm.

Proof. Let $\lambda_{i} \neq \mu$ for any $i$. Since $\mu$ is a standard right eigenvalue of $A+\Delta A$, there exists $0 \neq x \in \mathbb{H}^{n}$ such that $(A+\Delta A) x=x \mu$. This system is equivalent to the complex system

$$
\Psi_{A+\Delta A} \psi_{x}=\mu \psi_{x}
$$

which implies

$$
\left(\Psi_{A+\Delta A}-\mu I_{2 n}\right) \psi_{x}=0 .
$$

From this we can conclude that

$$
\left(\Psi_{A}+\Psi_{\Delta A}-\mu I_{2 n}\right) \psi_{x}=0 .
$$

The above system can be written as

$$
\left(\mu I_{2 n}-\Psi_{Y \Lambda Y^{-1}}\right) \psi_{x}=\Psi_{\Delta A} \psi_{x}
$$

and further as

$$
\Psi_{Y}\left(\mu I_{2 n}-\Psi_{\Lambda}\right) \Psi_{Y^{-1}} \psi_{x}=\Psi_{\Delta A} \psi_{x}
$$

Thus,

$$
\left(\mu I_{2 n}-\Psi_{\Lambda}\right)\left(\Psi_{Y}\right)^{-1} \psi_{x}=\left(\Psi_{Y}\right)^{-1} \Psi_{\Delta A} \psi_{x}
$$

which yields

$$
\left(\Psi_{Y}\right)^{-1} \psi_{x}=\left(\mu I_{2 n}-\Psi_{\Lambda}\right)^{-1}\left[\left(\Psi_{Y}\right)^{-1} \Psi_{\Delta A} \Psi_{Y}\right]\left(\Psi_{Y}\right)^{-1} \psi_{x} .
$$


Taking the matrix 2-norm (operator norm) on both sides, we get

$$
\begin{aligned}
\left\|\left(\Psi_{Y}\right)^{-1} \psi_{x}\right\|_{2} & \left.\leq\left\|\left(\mu I_{2 n}-\Psi_{\Lambda}\right)^{-1}\right\|_{2} \|\left(\Psi_{Y}\right)^{-1} \Psi_{\Delta A} \Psi_{Y}\right]\left\|_{2}\right\|\left(\Psi_{Y}\right)^{-1} \psi_{x} \|_{2}, \\
1 & \leq\left\|\left(\mu I_{2 n}-\Psi_{\Lambda}\right)^{-1}\right\|_{2}\left\|\left(\Psi_{Y}\right)^{-1}\right\|_{2}\left\|\Psi_{\Delta A}\right\|_{2}\left\|\Psi_{Y}\right\|_{2}, \\
1 & \leq \max _{\lambda_{i} \in \Lambda_{\mathbf{s}}(A)}\left\{\frac{1}{\left|\lambda_{i}-\mu\right|}, \frac{1}{\left|\overline{\lambda_{i}}-\mu\right|}\right\}\left\|\left(\Psi_{Y}\right)^{-1}\right\|_{2}\left\|\Psi_{\Delta A}\right\|_{2}\left\|\Psi_{Y}\right\|_{2} .
\end{aligned}
$$

From Lemma 3.1, we have

$$
\begin{aligned}
1 & \leq \max _{\lambda_{i} \in \Lambda_{\mathbf{s}}(A)}\left\{\frac{1}{\left|\lambda_{i}-\mu\right|}\right\}\left\|\left(\Psi_{Y}\right)^{-1}\right\|_{2}\left\|\Psi_{\Delta A}\right\|_{2}\left\|\Psi_{Y}\right\|_{2} \\
& \leq \frac{1}{\min _{\lambda_{i} \in \Lambda_{s}(A)}\left\{\left|\lambda_{i}-\mu\right|\right\}} \kappa\left(\Psi_{Y}\right)\left\|\Psi_{\Delta A}\right\|_{2} \\
& \leq \min _{\lambda_{i} \in \Lambda_{\mathbf{s}}(A)}\left\{\left|\lambda_{i}-\mu\right|\right\} \kappa\left(\Psi_{Y}\right)\left\|\Psi_{\Delta A}\right\|_{2} .
\end{aligned}
$$

From Lemma 2.6, we obtain

$$
\min _{\lambda_{i} \in \Lambda_{s}(A)}\left\{\left|\lambda_{i}-\mu\right|\right\} \leq \kappa(Y)\|\Delta A\|_{2}
$$

Lemma 3.3 yields

$$
\min _{\lambda_{i} \in \Lambda_{s}(A)}\left\{\left|\lambda_{i}-\mu\right|\right\}=\inf _{\eta_{j} \in \Lambda_{r}(A)}\left\{\left|\eta_{j}-\xi\right|\right\}
$$

where $\xi \in \Lambda_{r}(A+\Delta A)$. Hence,

$$
\inf _{\eta_{j} \in \Lambda_{r}(A)}\left\{\left|\eta_{j}-\xi\right|\right\} \leq \kappa(Y)\|\Delta A\|_{2}
$$

In particular, when $A \in M_{n}(\mathbb{H})$ is normal, Theorem 3.7 leads to the following corollary.

COROLlARY 3.8. Let $A \in M_{n}(\mathbb{H})$ be a normal matrix, and let $\mu$ be a standard right eigenvalue of the perturbed quaternionic matrix $A+\Delta A$. Then

$$
\operatorname{dist}\left(\mu, \Lambda_{s}(A)\right)=\min _{\lambda_{i} \in \Lambda_{s}(A)}\left\{\left|\lambda_{i}-\mu\right|\right\} \leq\|\Delta A\|_{2} .
$$

Moreover, we have

$$
\operatorname{dist}\left(\xi, \Lambda_{r}(A)\right)=\min _{\eta_{j} \in \Lambda_{r}(A)}\left\{\left|\eta_{j}-\xi\right|\right\} \leq\|\Delta A\|_{2},
$$

where $\xi \in \Lambda_{r}(A+\Delta A)$.

Many relative perturbation bounds for the eigenvalues of a nonsingular complex diagonalizable matrix have been proved in [8]. However, due to the non-commutativity of quaternions, the relative perturbation result [8, Corollary 2.2] is not easily extendable. Here, we extend the result [8, Corollary 2.2] from the complex field to the skew field of the quaternions by using the complex adjoint matrix of a quaternionic matrix and our results. We next derive the following theorem for a relative perturbation bound of the right eigenvalues.

THEOREM 3.9. Let $A \in M_{n}(\mathbb{H})$ be an invertible and diagonalizable matrix, that is, $A=Y \Lambda Y^{-1}$, where $Y \in M_{n}(\mathbb{H})$ is invertible and $\Lambda=\operatorname{diag}\left(\lambda_{1}, \ldots, \lambda_{n}\right)$ with $\lambda_{i}$ being the standard right eigenvalues of $A$. If $\mu$ is a standard right eigenvalue of $A+\Delta A$, then

$$
\operatorname{dist}\left(\mu, \Lambda_{s}(A)\right)=\min _{\lambda_{i} \in \Lambda_{s}(A)}\left\{\frac{\left|\lambda_{i}-\mu\right|}{\left|\lambda_{i}\right|}\right\} \leq \kappa(Y)\left\|A^{-1} \Delta A\right\|_{2} .
$$


Moreover, we have

$$
\operatorname{dist}\left(\xi, \Lambda_{r}(A)\right)=\inf _{\eta_{j} \in \Lambda_{r}(A)}\left\{\frac{\left|\eta_{j}-\xi\right|}{\left|\eta_{j}\right|}\right\} \leq \kappa(Y)\left\|A^{-1} \Delta A\right\|_{2},
$$

where $\xi \in \Lambda_{r}(A+\Delta A)$ and $\kappa(Y)=\|Y\|_{2}\left\|Y^{-1}\right\|_{2}$ is the condition number of $Y$ with respect to the matrix 2-norm.

Proof. Let $\lambda_{i} \neq \mu$ for any $i$. Since $\mu$ is a standard right eigenvalue of $A+\Delta A$, there exists $0 \neq x \in \mathbb{H}^{n}$ such that $(A+\Delta A) x=x \mu$. This system is equivalent to the complex system $\Psi_{A+\Delta A} \psi_{x}=\mu \psi_{x}$. Since $A$ is an invertible matrix, by multiplying with $-\Psi_{A^{-1}}$ from the left, we obtain

$$
\begin{aligned}
-\Psi_{A^{-1}}\left(\Psi_{A}+\Psi_{\Delta A}\right) \psi_{x} & =-\mu \Psi_{A^{-1}} \psi_{x}, \\
\left(-I_{2 n}-\Psi_{A^{-1} \Delta A}\right) \psi_{x} & =-\mu \Psi_{A^{-1}} \psi_{x}, \\
\left(\mu \Psi_{A^{-1}}-I_{2 n}\right) & =\Psi_{A^{-1} \Delta A} \psi_{x} .
\end{aligned}
$$

The matrix $A$ is diagonalizable, that is, $A=Y \Lambda Y^{-1}$, so

$$
\begin{aligned}
\left(\mu \Psi_{Y \Lambda^{-1} Y^{-1}}-I_{2 n}\right) \psi_{x} & =\Psi_{A^{-1} \Delta A} \psi_{x} \\
\Psi_{Y}\left(\mu \Psi_{\Lambda^{-1}}-I_{2 n}\right) \Psi_{Y^{-1}} \psi_{x} & =\Psi_{A^{-1} \Delta A} \psi_{x}
\end{aligned}
$$

After some manipulation, we get

$$
\left(\Psi_{Y}\right)^{-1} \psi_{x}=\left(\mu \Psi_{\Lambda^{-1}}-I_{2 n}\right)^{-1}\left[\left(\Psi_{Y}\right)^{-1} \Psi_{A^{-1} \Delta A} \Psi_{Y}\right]\left(\Psi_{Y}\right)^{-1} \psi_{x} .
$$

By applying the method of proof of Theorem 3.7, we have

$$
\operatorname{dist}\left(\mu, \Lambda_{s}(A)\right)=\min _{\lambda_{i} \in \Lambda_{s}(A)}\left\{\frac{\left|\lambda_{i}-\mu\right|}{\left|\lambda_{i}\right|}\right\} \leq \kappa(Y)\left\|A^{-1} \Delta A\right\|_{2},
$$

as well as

$$
\operatorname{dist}\left(\xi, \Lambda_{r}(A)\right)=\inf _{\eta_{j} \in \Lambda_{r}(A)}\left\{\frac{\left|\eta_{j}-\xi\right|}{\left|\eta_{j}\right|}\right\} \leq \kappa(Y)\left\|A^{-1} \Delta A\right\|_{2},
$$

where $\xi \in \Lambda_{r}(A+\Delta A)$.

Theorem 5.1 of [14] gives a universal eigenvalue bound. We extend this theorem from the complex field to the skew field of quaternions as follows.

THEOREM 3.10. Let $A \in M_{n}(\mathbb{H})$ be an invertible and diagonalizable matrix, that is, $A=Y \Lambda Y^{-1}$, where $Y \in M_{n}(\mathbb{H})$ is invertible and $\Lambda=\operatorname{diag}\left(\lambda_{1}, \ldots, \lambda_{n}\right)$ with $\lambda_{i}$ being the standard right eigenvalues of $A$. If $\mu$ is a standard right eigenvalue of the invertible quaternionic perturbed matrix $A+\Delta A$, then

$$
\min _{\lambda_{i} \in \Lambda_{s}(A)}\left\{\frac{\left|\lambda_{i}-\mu\right|}{\left|\lambda_{i}\right|^{p}|\mu|^{q}}\right\} \leq \kappa(Y)\left\|A^{-p} \Delta A(A+\Delta A)^{-q}\right\|_{2} .
$$

Moreover, we have

$$
\inf _{\eta_{j} \in \Lambda_{r}(A)}\left\{\frac{\left|\eta_{j}-\xi\right|}{\left|\eta_{i}\right|^{p}|\xi|^{q}}\right\} \leq \kappa(Y)\left\|A^{-p} \Delta A(A+\Delta A)^{-q}\right\|_{2},
$$

where $\xi \in \Lambda_{r}(A+\Delta A)$ and $\kappa(\cdot)$ is the condition number with respect to the matrix 2-norm. 
Proof. If $\mu \in \Lambda_{s}(A)$, then the result follows. We assume that $\mu \notin \Lambda_{s}(A)$. Since $\mu \notin \Lambda_{s}(A+\Delta A)$, there exists $0 \neq x \in \mathbb{H}^{n}$ such that $(A+\Delta A) x=x \mu$. This quaternion system is equivalent to the complex system

$$
\Psi_{(A+\Delta A)} \psi_{x}=\mu \Psi_{x}
$$

This implies

$$
\psi_{x}=-\left(\Psi_{A}-\mu I_{2 n}\right)^{-1} \Psi_{\Delta A} \psi_{x} .
$$

Let $p$ and $q$ be nonnegative integers including zero. Then

$$
\psi_{x}=-\left(\Psi_{A}-\mu I_{2 n}\right)^{-1}\left(\Psi_{A}\right)^{p}\left(\Psi_{A}\right)^{-p} \Psi_{\Delta A}\left(\Psi_{A+\Delta A}\right)^{-q}\left(\Psi_{A+\Delta A}\right)^{q} \psi_{x} .
$$

From $\Psi_{(A+\Delta A)} \psi_{x}=\mu \Psi_{x}$, we can easily see that $\left(\Psi_{A+\Delta A}\right)^{q} \psi_{x}=\mu^{q} \psi_{x}$. Thus,

$$
\psi_{x}=-\left[\mu^{-q}\left(\Psi_{A}\right)^{1-p}-\mu^{1-q}\left(\Psi_{A}\right)^{-p}\right]^{-1}\left(\Psi_{A}\right)^{-p} \Psi_{\Delta A}\left(\Psi_{A+\Delta A}\right)^{-q} \psi_{x} .
$$

Using $A=Y \Lambda Y^{-1}$, we have

$$
\psi_{x}=-\left[\mu^{-q}\left(\Psi_{Y \Lambda Y^{-1}}\right)^{1-p}-\mu^{1-q}\left(\Psi_{Y \Lambda Y^{-1}}\right)^{-p}\right]^{-1}\left(\Psi_{A}\right)^{-p} \Psi_{\Delta A}\left(\Psi_{A+\Delta A}\right)^{-q} \psi_{x} .
$$

From Theorem 2.5, we have

$$
\begin{aligned}
\left(\Psi_{Y \Lambda Y^{-1}}\right)^{1-p} & =\left(\Psi_{Y} \Psi_{\Lambda} \Psi_{Y^{-1}}\right)^{1-p} \\
& =\left(\Psi_{Y} \Psi_{\Lambda}\left(\Psi_{Y}\right)^{-1}\right)^{1-p}=\left(\Psi_{Y} \Psi_{\Lambda}\left(\Psi_{Y}\right)^{-1}\right)\left(\Psi_{Y} \Psi_{\Lambda}\left(\Psi_{Y}\right)^{-1}\right)^{-p} \\
& =\left(\Psi_{Y} \Psi_{\Lambda}\left(\Psi_{Y}\right)^{-1}\right)\left(\Psi_{Y}\left(\Psi_{\Lambda}\right)^{-1}\left(\Psi_{Y}\right)^{-1}\right)^{p} \\
& =\left(\Psi_{Y}\left(\Psi_{\Lambda}\right)^{-1}\left(\Psi_{Y}\right)^{-1}\right)^{p-1}\left(\Psi_{Y \Lambda Y^{-1}}\right)^{1-p}=\Psi_{Y}\left(\Psi_{\Lambda}\right)^{1-p}\left(\Psi_{Y}\right)^{-1} .
\end{aligned}
$$

Similarly, it follows that

$$
\left(\Psi_{Y \Lambda Y^{-1}}\right)^{-p}=\Psi_{Y}\left(\Psi_{\Lambda}\right)^{-p}\left(\Psi_{Y}\right)^{-1} .
$$

Thus, (3.4) yields

$$
\begin{gathered}
\psi_{x}=-\left[\mu^{-q} \Psi_{Y}\left(\Psi_{\Lambda}\right)^{1-p}\left(\Psi_{Y}\right)^{-1}\right. \\
\left.\quad-\mu^{1-q} \Psi_{Y}\left(\Psi_{\Lambda}\right)^{-p}\left(\Psi_{Y}\right)^{-1}\right]^{-1}\left(\Psi_{A}\right)^{-p} \Psi_{\Delta A}\left(\Psi_{A+\Delta A}\right)^{-q} \psi_{x}, \\
\psi_{x}=-\Psi_{Y}\left[\mu^{-q}\left(\Psi_{\Lambda}\right)^{1-p}-\mu^{1-q}\left(\Psi_{\Lambda}\right)^{-p}\right]^{-1}\left(\Psi_{Y}\right)^{-1} \Psi_{\left(A^{-p} \Delta A(A+\Delta A)^{-q}\right)} \psi_{x} .
\end{gathered}
$$

By taking the 2-norm on both sides, we obtain

$$
\begin{gathered}
\left\|\psi_{x}\right\|_{2} \leq\left\|\Psi_{Y}\right\|_{2}\left\|\left(\mu^{-q}\left(\Psi_{\Lambda}\right)^{1-p}-\mu^{1-q}\left(\Psi_{\Lambda}\right)^{-p}\right)^{-1}\right\|_{2} \times \\
\left\|\left(\Psi_{Y}\right)^{-1}\right\|_{2}\left\|\Psi_{\left(A^{-p} \Delta A(A+\Delta A)^{-q}\right)}\right\|_{2}\left\|\psi_{x}\right\|_{2}, \\
\text { 6) } 1 \leq\left\|\left(\mu^{-q}\left(\Psi_{\Lambda}\right)^{1-p}-\mu^{1-q}\left(\Psi_{\Lambda}\right)^{-p}\right)^{-1}\right\|_{2} \kappa(Y)\left\|\left(A^{-p} \Delta A(A+\Delta A)^{-q}\right)\right\|_{2} .
\end{gathered}
$$


Further, we have

$$
\begin{aligned}
& \left\|\left(\mu^{-q}\left(\Psi_{\Lambda}\right)^{1-p}-\mu^{1-q}\left(\Psi_{\Lambda}\right)^{-p}\right)^{-1}\right\|_{2} \\
& =\max _{\lambda_{i} \in \Lambda_{s}(A)}\left\{\frac{1}{\left|\mu^{-q} \lambda_{i}^{1-p}-\mu^{1-q} \lambda_{i}^{-p}\right|}, \frac{1}{\left|\mu^{-q}\left(\overline{\lambda_{i}}\right)^{1-p}-\mu^{1-q}\left(\overline{\lambda_{i}}\right)^{-p}\right|}\right\} \\
& \quad=\max _{\lambda_{i} \in \Lambda_{s}(A)}\left\{\frac{\left|\lambda_{i}\right|^{p}|\mu|^{q}}{\left|\lambda_{i}-\mu\right|}, \frac{\left|\overline{\lambda_{i}}\right|^{p}|\mu|^{q}}{\left|\overline{\lambda_{i}}-\mu\right|}\right\} .
\end{aligned}
$$

From Lemma 3.1, we obtain

$$
\left\|\left(\mu^{-q}\left(\Psi_{\Lambda}\right)^{1-p}-\mu^{1-q}\left(\Psi_{\Lambda}\right)^{-p}\right)^{-1}\right\|_{2}=\max _{\lambda_{i} \in \Lambda_{s}(A)} \frac{\left|\lambda_{i}\right|^{p}|\mu|^{q}}{\left|\lambda_{i}-\mu\right|}=\frac{1}{\min _{\lambda_{i} \in \Lambda_{s}(A) \frac{\left|\lambda_{i}-\mu\right|}{\left|\lambda_{i}\right|^{p}|\mu|^{q}}}} .
$$

Now, (3.6) gives the inequality

$$
\min _{\lambda_{i} \in \Lambda_{s}(A)}\left\{\frac{\left|\lambda_{i}-\mu\right|}{\left|\lambda_{i}\right|^{p}|\mu|^{q}}\right\} \leq \kappa(Y)\left\|A^{-p} \Delta A(A+\Delta A)^{-q}\right\|_{2} .
$$

As we have already seen,

$$
\min _{\lambda_{i} \in \Lambda_{s}(A)}\left\{\left|\lambda_{i}-\mu\right|\right\}=\inf _{\eta_{j} \in \Lambda_{r}(A)}\left\{\left|\eta_{j}-\xi\right|\right\}
$$

where $\xi \in \Lambda_{r}(A+\Delta A)$. Thus, we arrive at the desired result

$$
\inf _{\eta_{j} \in \Lambda_{r}(A)}\left\{\frac{\left|\eta_{j}-\xi\right|}{\left|\eta_{i}\right|^{p}|\xi|^{q}}\right\} \leq \kappa(Y)\left\|A^{-p} \Delta A(A+\Delta A)^{-q}\right\|_{2} .
$$

Some special cases of Theorem 3.10 give several bounds for the right eigenvalues:

(i) Assume $p=q=0$. Then Theorem 3.10 is identical to Theorem 3.7 (Bauer-Fike-type theorem).

(ii) Assume $p=1, q=0$. Then Theorem 3.10 is identical to Theorem 3.9 (relative perturbation bound).

(iii) Assume $p=0, q=1$. Then Theorem 3.10 gives a new relative perturbation bound, that is,

$$
\min _{\lambda_{i} \in \Lambda_{s}(A)}\left\{\frac{\left|\lambda_{i}-\mu\right|}{|\mu|}\right\} \leq \kappa(Y)\left\|\Delta A(A+\Delta A)^{-1}\right\|_{2}
$$

and

$$
\inf _{\eta_{j} \in \Lambda_{r}(A)}\left\{\frac{\left|\eta_{j}-\xi\right|}{|\xi|}\right\} \leq \kappa(Y)\left\|\Delta A(A+\Delta A)^{-1}\right\|_{2}
$$

It is clear that the perturbed right eigenvalue is in the denominator.

We discuss a localization theorem for the standard right eigenvalues of the perturbed quaternionic matrix $A+\Delta A$. We will see that a right (nonstandard) eigenvalue of the perturbed quaternionic matrix $A+\Delta A$ is not necessarily contained in the union of $n$ discs

$$
\Omega_{i}(A)=\left\{z \in \mathbb{C}:\left|z-\lambda_{i}\right| \leq \kappa(Y)\|\Delta A\|_{2}\right\}, \quad i=1,2, \ldots, n,
$$


where $A$ is diagonalizable, that is, $A=Y \operatorname{diag}\left(\lambda_{i}\right) Y^{-1}$ and $\lambda_{i} \in \Lambda_{s}(A)$. For example, let

$$
A=\left[\begin{array}{cc}
1+\mathbf{i} & 0 \\
0 & \mathbf{i}
\end{array}\right], \quad \Delta A=\left[\begin{array}{ll}
\epsilon & 0 \\
0 & \epsilon
\end{array}\right] .
$$

Then

$$
A+\Delta A=\left[\begin{array}{cc}
1+\epsilon+\mathbf{i} & 0 \\
0 & \mathbf{i}+\epsilon
\end{array}\right], \quad\|\Delta A\|_{2}=\epsilon
$$

Since $A$ is normal, $\kappa(Y)=1$. We set $\epsilon=10^{-3}$. From (3.7), we get the following two discs:

$$
\Omega_{1}(A)=\left\{z \in \mathbb{C}:|z-1-\mathbf{i}| \leq 10^{-3}\right\}, \quad \Omega_{2}(A)=\left\{z \in \mathbb{C}:|z-\mathbf{i}| \leq 10^{-3}\right\} .
$$

The perturbed matrix $A+\Delta A$ has two standard right eigenvalues, $1+\epsilon+\mathbf{i}$ and $\mathbf{i}+\epsilon$. However, $1+\epsilon-\mathbf{i}$ is also a right eigenvalue of $A+\Delta A$, but it is not contained in any of these discs. Fortunately, from Theorem 3.7, all the standard right eigenvalues of $A+\Delta A$ are contained in the union of the $n$ discs (3.7), that is,

$$
\Lambda_{s}(A+\Delta A) \subseteq \Omega(A)=\bigcup_{i=1}^{n} \Omega_{i}(A) .
$$

The following result is a sufficient condition for the stability of the perturbed quaternionic matrix $A+\triangle A$.

Proposition 3.11. Let $A \in M_{n}(\mathbb{H})$ be a diagonalizable matrix, that is, $A=Y \Lambda Y^{-1}$, where $Y \in M_{n}(\mathbb{H})$ is invertible and $\Lambda=\operatorname{diag}\left(\lambda_{1}, \ldots, \lambda_{n}\right)$ with $\lambda_{i}$ being the standard right eigenvalues of $A$. Assume that

$$
\Re\left(\lambda_{i}\right)+\kappa(Y)\|\Delta A\|_{2}<0, \quad i=1,2, \ldots, n .
$$

Then the perturbed quaternionic matrix $A+\Delta A$ is stable.

Proof. Let $\mu$ be any standard right eigenvalue of $A+\Delta A$. Then from (3.7), we have $\mu \in \bigcup_{i=1}^{n} \Omega_{i}(A)$. Without loss of generality, we may assume $\mu \in \Omega_{l}(A)$, that is,

$$
\left|\mu-\lambda_{l}\right| \leq \kappa(Y)\|\Delta A\|_{2} .
$$

Consider $\mu=\mu_{1}+\mu_{2} \mathbf{i}$ and $\lambda_{l}=\lambda_{1}+\lambda_{2} \mathbf{i}$. Now, from (3.8), we obtain

$$
\left|\left(\mu_{1}-\lambda_{1}\right)+\left(\mu_{2}-\lambda_{2}\right) \mathbf{i}\right| \leq-\Re\left(\lambda_{l}\right)=-\lambda_{1} .
$$

The inequality in (3.9) can only be satisfied when $\mu_{1}<0$, that is, $\Re(\mu)<0$. Since $\Re(\mu)=\operatorname{real}\left(\rho^{-1} \mu \rho\right)$ for all $\rho \in \mathbb{H} \backslash\{0\}$, that is, the real part of the standard right eigenvalue $\mu$ and the real part of the corresponding nonstandard right eigenvalues are the same. Thus, $\rho^{-1} \mu \rho \in \mathbb{H}^{-}$. This shows that the matrix $A+\Delta A$ is stable.

Let $A \in M_{n}(\mathbb{H})$ be a central closed matrix. Then, by Theorem 3.7, we obtain

$$
\min _{\lambda_{i} \in \Lambda_{\mathbf{s}}(A)}\left\{\left|\lambda_{i}-\mu\right|\right\} \leq \kappa(Y)\|\Delta A\|_{2},
$$

where $\lambda_{i}$ are the real standard right eigenvalues of $A$, and $\mu$ is a standard right eigenvalue of $A+\Delta A$. Since $\mu$ is a standard right eigenvalue of $A+\Delta A$, there exists $\rho \in \mathbb{H} \backslash\{0\}$ such that $\rho^{-1} \mu \rho=\xi$ is also a right eigenvalue of $A+\Delta A$ and

$$
\left|\lambda_{i}-\mu\right|=\left|\lambda_{i}-\rho \xi \rho^{-1}\right|=\left|\rho\left(\lambda_{i}-\xi\right) \rho^{-1}\right|=\left|\lambda_{i}-\xi\right|, \quad i=1,2, \ldots, n .
$$


Thus, we have

$$
\min _{\lambda_{i} \in \Lambda_{\mathbf{r}}(A)}\left\{\left|\lambda_{i}-\xi\right|\right\} \leq \kappa(Y)\|\Delta A\|_{2}
$$

From inequality (3.10), we can see that all right eigenvalues of $A+\Delta A$ are contained in the union of $n$ balls,

$$
\mathcal{G}_{i}(A)=\left\{z \in \mathbb{H}:\left|z-\lambda_{i}\right| \leq \kappa(Y)\|\Delta A\|_{2}\right\}, \quad i=1,2, \ldots, n,
$$

that is,

$$
\Lambda_{r}(A+\Delta A) \subseteq \mathcal{G}(A)=\bigcup_{i=1}^{n} \mathcal{G}_{i}(A)
$$

An interesting result in the perturbation theory in terms of residual vectors states the following [13, Theorem 6.3.14]: if $A \in M_{n}(\mathbb{C})$ is diagonalizable with $A=Y \Lambda Y^{-1}$, where $\Lambda=\operatorname{diag}\left(\lambda_{1}, \ldots, \lambda_{n}\right)$, and $\tilde{x} \in \mathbb{C}^{n}$ is a given nonzero vector with residual vector $r=A \tilde{x}-\tilde{\lambda} \tilde{x}$ for some given $\tilde{\lambda} \in \mathbb{C}$, then there is an eigenvalue $\lambda_{i}$ of $A$ for which

$$
\left|\tilde{\lambda}-\lambda_{i}\right| \leq \kappa(Y) \frac{\|r\|_{2}}{\|\tilde{x}\|_{2}} .
$$

Several bounds follow as special cases of (3.11):

Case (i): If $A$ is normal, then there is some eigenvalue $\lambda_{i}$ of $A$ for which

$$
\left|\tilde{\lambda}-\lambda_{i}\right| \leq \frac{\|r\|_{2}}{\|\tilde{x}\|_{2}}
$$

Case (ii): If $A$ is Hermitian and $\tilde{\lambda}$ is a given real number. Then for $\|\tilde{x}\|_{2}=1$, we have

$$
\left|\tilde{\lambda}-\lambda_{i}\right| \leq\|r\|_{2} .
$$

Since real numbers commute with quaternions, a real left eigenvalue of a matrix $A \in M_{n}(\mathbb{H})$ is also a right eigenvalue of $A$ and vice versa. By applying this argument, we extend the result (3.12) from the complex field to the skew field of quaternions as follows.

TheOREM 3.12. Let $A \in M_{n}(\mathbb{H})$ be a Hermitian matrix. For some $\tilde{\mu} \in \mathbb{R}$ and $\tilde{x} \in \mathbb{H}^{n}$ with $\|\tilde{x}\|_{2}=1$, define the residual vector $r=A \tilde{x}-\tilde{\mu} \tilde{x}$. Then $|\tilde{\mu}-\mu| \leq\|r\|_{2}$ for some $\mu \in \Lambda_{r}(A)$.

Proof. Since $\mu \in \Lambda_{r}(A)$, we also have $\mu \in \Lambda_{l}(A)$. Now if $\tilde{\mu} \notin \Lambda_{r}(A)$, then $\tilde{\mu} \notin \Lambda_{l}(A)$. Hence $\left(A-\tilde{\mu} I_{n}\right)^{-1}$ exists, so we can write $r=A \tilde{x}-\tilde{\mu} \tilde{x}$ as

$$
\tilde{x}=\left(A-\tilde{\mu} I_{n}\right)^{-1} r .
$$

Since $A$ is a Hermitian matrix, by Theorem $2.11, A$ is unitarily diagonalizable, that is, $V^{-1} A V=\operatorname{diag}\left(\mu_{i}\right), i=1,2, \ldots, n$, for some quaternionic unitary matrix $V$. Now, by applying the method of proof of Theorem 3.7, we have the desired result

$$
\min _{\mu_{i} \in \Lambda_{\mathbf{s}}(A)}\left\{\left|\tilde{\mu}-\mu_{i}\right|\right\} \leq\|r\|_{2} .
$$


3.2. Right eigenvalue perturbation bounds for non-diagonalizable quaternionic matrices. Perturbation bounds for the eigenvalues of a complex matrix in the non-diagonalizable case as well as the block diagonal decomposition of a complex matrix and Jordan blocks have been studied in $[5,10]$. We extend the perturbation result $[5$, Theorem 2] to the quaternionic case via the block diagonal decomposition of quaternionic matrices.

Theorem 3.13. Let $A \in M_{n}(\mathbb{H})$. Consider $A=Y T Y^{-1}, T=\operatorname{diag}\left(V_{1}, \ldots, V_{k}\right)$, where $V_{i}=\Lambda_{i}+N_{i} \in M_{n_{i}}(\mathbb{H})$ is upper triangular, $\Lambda_{i}$ is diagonal, and $N_{i}$ is strict upper triangular, for $i=1,2, \ldots, k$. If $\mu$ is a standard right eigenvalue of $A+\Delta A$, then there exists $\lambda_{j} \in \Lambda_{s}(A)$ such that

$$
\left|\lambda_{j}-\mu\right| \leq \max \left(\chi, \chi^{1 / n_{j}}\right),
$$

where $\chi=\left\|Y^{-1} \Delta A Y\right\|_{2} \sum_{t=0}^{n_{j}-1}\left\|N_{j}\right\|_{2}^{t}$ and $N_{j}^{n_{j}}=0_{n_{j}}$ with $N_{j}^{n_{j}-1} \neq 0_{n_{j}}$.

Proof. Let $\mu \notin \Lambda_{s}(A)$. Since $\mu$ is a standard right eigenvalue of $A+\Delta A$, there exists $0 \neq x \in \mathbb{H}^{n}$ such that $(A+\Delta A) x=x \mu$. Then, following the method of proof of Theorem 3.7, we get

$$
1 \leq\left\|\left(\mu I_{2 n}-\Psi_{T}\right)^{-1}\right\|_{2}\left\|Y^{-1} \Delta A Y\right\|_{2}
$$

We have that

$$
\frac{1}{\left\|\left(\mu I_{2 n}-\Psi_{T}\right)^{-1}\right\|_{2}}=\frac{1}{\max _{1 \leq i \leq k}\left\|\left(\mu I_{2 n_{i}}-\Psi_{V_{i}}\right)^{-1}\right\|_{2}}=\frac{1}{\left\|\left(\mu I_{2 n_{j}}-\Psi_{V_{j}}\right)^{-1}\right\|_{2}} .
$$

Define

$$
\tau=\frac{1}{\left\|\left(\mu I_{2 n_{j}}-\Psi_{\Lambda_{j}}\right)^{-1}\right\|_{2}}=\frac{1}{\max \left\{\frac{1}{\left|\mu-\lambda_{j}\right|}, \frac{1}{\left|\mu-\overline{\lambda_{j}}\right|}\right\}}=\left|\mu-\lambda_{j}\right| .
$$

Since $N_{j}^{n_{j}}=0$, from Lemma 3.4, we obtain $\Psi_{N_{j}}^{n_{j}}=0$. As $N_{j}$ is a strict upper triangular quaternionic matrix, $\Psi_{N_{j}}$ is also strict upper triangular. Hence,

$$
\left(\mu I_{2 n_{j}}-\Psi_{\Lambda_{j}}\right) \Psi_{N_{j}}=\Psi_{N_{j}}\left(\mu I_{2 n_{j}}-\Psi_{\Lambda_{j}}\right) .
$$

Therefore,

$$
\left[\left(\mu I_{2 n_{j}}-\Psi_{\Lambda_{j}}\right)^{-1} \Psi_{N_{j}}\right]^{n_{j}}=0 .
$$

Consequently, we get

$$
\left(\mu I_{2 n_{j}}-\Psi_{V_{j}}\right)^{-1}=\sum_{t=0}^{n_{j}-1}(-1)^{t}\left[\left(\mu I_{2 n_{j}}-\Psi_{\Lambda_{j}}\right)^{-1} \Psi_{N_{j}}\right]^{t}\left(\mu I_{2 n_{j}}-\Psi_{\Lambda_{j}}\right)^{-1}
$$

Thus, we have

$$
\left\|\left(\mu I_{2 n}-\Psi_{T}\right)^{-1}\right\|_{2}=\left\|\left(\mu I_{2 n_{j}}-\Psi_{V_{j}}\right)^{-1}\right\|_{2} \leq \frac{1}{\tau} \sum_{t=0}^{n_{j}-1}\left[\frac{\left\|N_{j}\right\|_{2}}{\tau}\right]^{t} .
$$

If $\tau>1$, then

$$
\left\|\left(\mu I_{2 n_{j}}-\Psi_{V_{j}}\right)^{-1}\right\|_{2} \leq \frac{1}{\tau} \sum_{t=0}^{n_{j}-1}\left\|N_{j}\right\|_{2}^{t}
$$


From (3.13), (3.14), and (3.15), we obtain

$$
1 \leq \frac{1}{\tau} \sum_{t=0}^{n_{j}-1}\left\|N_{j}\right\|_{2}^{t}\left(\left\|Y^{-1} \Delta A Y\right\|_{2}\right) .
$$

Let $\chi=\left\|Y^{-1} \Delta A Y\right\|_{2} \sum_{t=0}^{n_{j}-1}\left\|N_{j}\right\|_{2}^{t}$. Then, $\tau \leq \chi$. If $\tau \leq 1$, then

$$
\left\|\left(\mu I_{2 n}-\Psi_{T}\right)^{-1}\right\|_{2}=\left\|\left(\mu I_{2 n_{j}}-\Psi_{V_{j}}\right)^{-1}\right\|_{2} \leq \frac{1}{\tau^{n_{j}}} \sum_{t=0}^{n_{j}-1}\left\|N_{j}\right\|_{2}^{t} .
$$

Hence, (3.13), (3.14), and (3.16) yield

$$
1 \leq \frac{1}{\tau^{n_{j}}} \sum_{t=0}^{n_{j}-1}\left\|N_{j}\right\|_{2}^{t}\left(\left\|Y^{-1} \Delta A Y\right\|_{2}\right) .
$$

Thus, $\tau^{n_{j}} \leq \chi$, and hence, $\tau \leq \chi^{n_{j}}$. From the above, it is clear that $\left|\lambda_{j}-\mu\right| \leq \max \left(\chi, \chi^{1 / n_{j}}\right)$, where

$$
\chi=\left\|Y^{-1} \Delta A Y\right\|_{2} \sum_{t=0}^{n_{j}-1}\left\|N_{j}\right\|_{2}^{t} .
$$

By taking an idea of [10, Corollary 1], we present a perturbation result for quaternionic matrices via the Jordan canonical form of a quaternionic matrix.

THEOREM 3.14. Let $A \in M_{n}(\mathbb{H})$ with $Y^{-1} A Y=J=\operatorname{diag}\left(J_{m_{i}}\left(\lambda_{i}\right)\right)$, where $J_{m_{i}}\left(\lambda_{i}\right)(1 \leq i \leq t)$ are the Jordan blocks of $A$. Let $\Delta A \in M_{n}(\mathbb{H})$. If $\mu$ is a standard right eigenvalue of $A+\Delta A$, then

$$
\min _{1 \leq i \leq t}\left\{\frac{1}{\left\|\left(J_{m_{i}}\left(\lambda_{i}\right)-\mu I_{m_{i}}\right)^{-1}\right\|_{2}}, \frac{1}{\left\|\left(\overline{J_{m_{i}}\left(\lambda_{i}\right)}-\mu I_{m_{i}}\right)^{-1}\right\|_{2}}\right\} \leq \kappa(Y)\|\Delta A\|_{2},
$$

where $\kappa(\cdot)$ is the condition number with respect to the matrix 2-norm.

Proof. If $\mu$ is not a standard right eigenvalue of any of the Jordan block matrices $J_{m_{i}}\left(\lambda_{i}\right)$, then the statement is nontrivial. Since $\mu$ is a standard right eigenvalue of $A+\Delta A$, there exists $0 \neq x \in \mathbb{H}^{n}$ such that $(A+\Delta A) x=x \mu$, and this system is equivalent to the complex system

$\Psi_{A+\Delta A} \psi_{x}=\mu \psi_{x} \quad \Leftrightarrow \quad\left(\Psi_{A+\Delta A}-\mu I_{2 n}\right) \psi_{x}=0 \quad \Leftrightarrow \quad\left(\Psi_{A}+\Psi_{\Delta A}-\mu I_{2 n}\right) \psi_{x}=0$.

Thus, $A$ has a Jordan canonical form via the invertible quaternionic matrix $Y$. Hence,

$$
\begin{aligned}
\left(\mu I_{2 n}-\Psi_{Y \operatorname{diag}\left(J_{m_{i}}\left(\lambda_{i}\right)\right) Y^{-1}}\right) \psi_{x} & =\Psi_{\Delta A} \psi_{x}, \\
\Psi_{Y}\left(\mu I_{2 n}-\Psi_{\operatorname{diag}\left(J_{m_{i}}\left(\lambda_{i}\right)\right)}\right) \Psi_{Y^{-1}} \psi_{x} & =\Psi_{\Delta A} \psi_{x}, \\
\left(\mu I_{2 n}-\Psi_{\operatorname{diag}\left(J_{m_{i}}\left(\lambda_{i}\right)\right)}\right)\left(\Psi_{Y}\right)^{-1} \psi_{x} & =\left(\Psi_{Y}\right)^{-1} \Psi_{\Delta A} \psi_{x} .
\end{aligned}
$$

This implies that

$$
\left(\Psi_{Y}\right)^{-1} \psi_{x}=\left(\mu I_{2 n}-\Psi_{\operatorname{diag}\left(J_{m_{i}}\left(\lambda_{i}\right)\right)}\right)^{-1}\left[\left(\Psi_{Y}\right)^{-1} \Psi_{\Delta A} \Psi_{Y}\right]\left(\Psi_{Y}\right)^{-1} \psi_{x} .
$$


Taking the matrix 2-norm on both sides of the above equation, we obtain

$$
\begin{aligned}
&\left\|\left(\Psi_{Y}\right)^{-1} \psi_{x}\right\|_{2} \leq\left\|\left(\mu I_{2 n}-\Psi_{\operatorname{diag}\left(J_{m_{i}}\left(\lambda_{i}\right)\right)}\right)^{-1}\right\|_{2}\left\|\left[\left(\Psi_{Y}\right)^{-1} \Psi_{\Delta A} \Psi_{Y}\right]\right\|_{2}\left\|\left(\Psi_{Y}\right)^{-1} \psi_{x}\right\|_{2} \\
& 1 \leq\left\|\left(\mu I_{2 n}-\Psi_{\operatorname{diag}\left(J_{m_{i}}\left(\lambda_{i}\right)\right)}\right)^{-1}\right\|_{2}\left\|\left(\Psi_{Y}\right)^{-1}\right\|_{2}\left\|\Psi_{\Delta A}\right\|_{2}\left\|\Psi_{Y}\right\|_{2}, \\
& 1 \leq \max _{1 \leq i \leq t}\left\{\left\|\left(J_{m_{i}}\left(\lambda_{i}\right)-\mu I_{m_{i}}\right)^{-1}\right\|_{2},\left\|\left(\overline{J_{m_{i}}\left(\lambda_{i}\right)}-\mu I_{m_{i}}\right)^{-1}\right\|_{2}\right\} \times \\
&\left\|\left(\Psi_{Y}\right)^{-1}\right\|_{2}\left\|\Psi_{\Delta A}\right\|_{2}\left\|\Psi_{Y}\right\|_{2}, \\
& 1 \leq \max _{1 \leq i \leq t}\left\{\left\|\left(J_{m_{i}}\left(\lambda_{i}\right)-\mu I_{m_{i}}\right)^{-1}\right\|_{2},\left\|\left(\overline{J_{m_{i}}\left(\lambda_{i}\right)}-\mu I_{m_{i}}\right)^{-1}\right\|_{2}\right\} \times \\
&\left\|\left(\Psi_{Y}\right)^{-1}\right\|_{2}\left\|\Psi_{\Delta A}\right\|_{2}\left\|\Psi_{Y}\right\|_{2} .
\end{aligned}
$$

The above inequality can be rewritten as

$$
\begin{aligned}
& \frac{1}{\max _{1 \leq i \leq t}\left\{\left\|\left(J_{m_{i}}\left(\lambda_{i}\right)-\mu I_{m_{i}}\right)^{-1}\right\|_{2},\left\|\left(\overline{J_{m_{i}}\left(\lambda_{i}\right)}-\mu I_{m_{i}}\right)^{-1}\right\|_{2}\right\}} \\
& \leq\left\|\left(\Psi_{Y}\right)^{-1}\right\|_{2}\left\|\Psi_{\Delta A}\right\|_{2}\left\|\Psi_{Y}\right\|_{2},
\end{aligned}
$$

that is,

$$
\min _{1 \leq i \leq t}\left\{\frac{1}{\left\|\left(J_{m_{i}}\left(\lambda_{i}\right)-\mu I_{m_{i}}\right)^{-1}\right\|_{2}}, \frac{1}{\left\|\left(\overline{J_{m_{i}}\left(\lambda_{i}\right)}-\mu I_{m_{i}}\right)^{-1}\right\|_{2}}\right\} \leq \kappa\left(\Psi_{Y}\right)\left\|\Psi_{\Delta A}\right\|_{2} .
$$

Then, from Lemma 2.6, we have

$$
\min _{1 \leq i \leq t}\left\{\frac{1}{\left\|\left(J_{m_{i}}\left(\lambda_{i}\right)-\mu I_{m_{i}}\right)^{-1}\right\|_{2}}, \frac{1}{\left\|\left(\overline{J_{m_{i}}\left(\lambda_{i}\right)}-\mu I_{m_{i}}\right)^{-1}\right\|_{2}}\right\} \leq \kappa(Y)\|\Delta A\|_{2} .
$$

From Theorem 3.14, we conclude that all the standard right eigenvalues of the perturbed quaternionic matrix $A+\Delta A$ (where $A$ is not necessarily a diagonalizable matrix) are contained in the union of $t$ sets $P_{i}(A)=T_{i}(A) \cup K_{i}(A), i=1, \ldots, t$, where

$$
\begin{aligned}
T_{i}(A) & =\left\{z \in \mathbb{C}:\left\|\left(J_{m_{i}}\left(\lambda_{i}\right)-\mu I_{m_{i}}\right)^{-1}\right\|_{2}^{-1} \leq \kappa(Y)\|\Delta A\|_{2}\right\}, \\
K_{i}(A) & =\left\{z \in \mathbb{C}:\left\|\left(\overline{J_{m_{i}}\left(\lambda_{i}\right)}-\mu I_{m_{i}}\right)^{-1}\right\|_{2}^{-1} \leq \kappa(Y)\|\Delta A\|_{2}\right\},
\end{aligned}
$$

that is,

$$
\Lambda_{s}(A+\Delta A) \subseteq P(A)=\left(\bigcup_{i=1}^{t} T_{i}(A)\right) \cup\left(\bigcup_{i=1}^{t} K_{i}(A)\right) .
$$

REMARK 3.15. From Lemma 2.7 it follows that all results for the 2-norm hold for the Frobenius norm as well.

4. Perturbation bounds for the zeros of quaternionic polynomials. The companion matrices of the simple monic polynomials $p_{l}(z)$ and $p_{r}(z)$ defined by (1.2) and (1.3), respectively, are given by

$$
C_{p_{l}}=\left[\begin{array}{cccc}
0 & 1 & & 0 \\
\vdots & & \ddots & \\
0 & 0 & & 1 \\
-q_{0} & -q_{1} & \ldots & -q_{m-1}
\end{array}\right], \quad C_{p_{r}}=C_{p_{l}}^{T}
$$


We need the following results.

Proposition 4.1 ([29, Proposition 1]). Let $\lambda \in \mathbb{H}$. Then $\lambda$ is a zero of the monic polynomial $p_{l}(z)$ if and only if $\lambda$ is a left eigenvalue of its corresponding companion matrix $C_{p_{l}}$.

Analogous to Proposition 4.1, the following result is presented for $p_{r}(z)$.

PROPOSITION 4.2. Let $\lambda \in \mathbb{H}$. Then $\lambda$ is a zero of the monic polynomial $p_{r}(z)$ if and only if $\lambda$ is a left eigenvalue of its corresponding companion matrix $C_{p_{r}}$.

It is shown in [29, Corollary 1] that if $\lambda$ is a left eigenvalue of $C_{p_{l}}$, then $\lambda$ is also a right eigenvalue of $C_{p_{l}}$. Hence, all the zeros of the polynomial $p_{l}(z)$ are right eigenvalues of $C_{p_{l}}$. However, a right eigenvalue is not necessarily the zero of the polynomial $p_{l}(z)$. For example, take a simple monic polynomial $p_{l}(z)=z^{2}+\mathbf{j} z+2$. Its companion matrix is

$$
C_{p_{l}}=\left[\begin{array}{cc}
0 & 1 \\
-2 & -\mathbf{j}
\end{array}\right] .
$$

Here $\mathbf{i}$ is a right eigenvalue of $C_{p_{l}}$, however, $\mathbf{i}$ is not a zero of $p_{l}(z)$.

COROLlary 4.3 ([29, Corollary 1]). If $\lambda$ is a left eigenvalue of the companion matrix $C_{p_{l}}$, then it is also a right eigenvalue.

THEOREM 4.4. Let $p_{l}(z)=z^{m}+\sum_{k=0}^{m-1} q_{k} z^{k}$ be a quaternionic simple monic polynomial. Let $C_{p_{l}}=Y \operatorname{diag}\left(V_{1}, V_{2}, \ldots, V_{t}\right) Y^{-1}$ with $V_{i}=\Lambda_{i}+N_{i} \in M_{n_{i}}(\mathbb{H})$ being upper triangular, $\Lambda_{i}$ diagonal, and $N_{i}$ strict upper triangular, for $i=1,2, \ldots, t$. Assume that $\widehat{p_{l}}(z)=z^{m}+\sum_{k=0}^{m-1} \widehat{q_{k}} z^{k}$ is a perturbation of $p_{l}(z)$ with $\widehat{q_{k}}=q_{k}+\Delta q_{k}$, $\left|\Delta q_{k}\right| \leq \epsilon, k=0,1, \ldots, m-1$. Let $e_{m}=[0, \ldots, 0,1]^{T} \in \mathbb{R}^{m}$. Then for any complex zero $\widehat{z_{k}} \in Z_{\mathbb{C}}\left(\widehat{p_{l}}(z)\right)$, there exists a complex zero $z_{j} \in Z_{\mathbb{C}}\left(p_{l}(z)\right)$ such that

$$
\left|\widehat{z_{k}}-z_{j}\right| \leq \max \left(\chi, \chi^{1 / n_{j}}\right),
$$

where

$$
\chi=\left\|Y^{-1} \Delta C_{p_{l}} Y\right\|_{2} \sum_{\eta=0}^{n_{j}-1}\left\|N_{j}\right\|_{2}^{\eta}, \quad \Delta C_{p_{l}}=-e_{m}\left[\Delta q_{0}, \ldots, \Delta q_{m-1}\right] .
$$

Proof. Let us consider the companion matrix $C_{p_{l}}$ corresponding to the simple monic polynomial $p_{l}(z)$ such that $C_{p_{l}}=Y \operatorname{diag}\left(V_{1}, V_{2}, \ldots, V_{t}\right) Y^{-1}$. From the definition of the matrix $\Delta C_{p_{l}}=-e_{m}\left[\Delta q_{0}, \ldots, \Delta q_{m-1}\right]$, it follows that

$$
C_{p_{l}}+\Delta C_{p_{l}}=\left[\begin{array}{ccccc}
0 & 1 & 0 & \ldots & 0 \\
0 & 0 & 1 & & 0 \\
\vdots & \vdots & \vdots & \ddots & \vdots \\
0 & 0 & 0 & \ldots & 1 \\
-\widehat{q_{0}} & -\widehat{q_{1}} & -\widehat{q_{2}} & \ldots & -\widehat{q_{m-1}}
\end{array}\right] .
$$

It is known that the left eigenvalues of $C_{p_{l}}$ and the zeros of $p_{l}(z)$ are the same. Also, the left spectrum of $C_{p_{l}}$ falls into the right spectrum of $C_{p_{l}}$. Theorem 3.13 gives the perturbation bound for the standard right eigenvalues of a quaternionic matrix and a perturbed quaternionic matrix. As we know that standard right eigenvalues of a quaternionic matrix are complex numbers, by the method of proof of Theorem 3.13, we get the desired result. 
THEOREM 4.5. Let $p_{l}(z)=z^{m}+\sum_{k=0}^{m-1} q_{k} z^{k}$ be a quaternionic simple monic polynomial with its companion matrix $C_{p_{l}}=Y D Y^{-1}, D=\operatorname{diag}\left(\lambda_{1}, \ldots, \lambda_{m}\right), \lambda_{t} \in \Lambda_{s}(A)$, $t=1,2, \ldots, m$. Assume that $\widehat{p}_{l}(z)=z^{m}+\sum_{k=0}^{m-1} \widehat{q}_{k} z^{k}$ is a perturbation of $p_{l}(z)$ with $\widehat{q_{k}}=q_{k}+\Delta q_{k},\left|\Delta q_{k}\right| \leq \epsilon,(0 \leq k \leq m-1)$. Then the zeros of $p_{l}(z)$ and $\widehat{p_{l}}(z)$ are given by

$$
\inf _{\substack{z_{i} \in Z_{\mathbb{H}}\left(\widehat{p_{l}}(z)\right) \\ z_{j} \in Z_{\mathbb{H}}\left(p_{l}(z)\right)}}\left|\widehat{z_{i}}-z_{j}\right| \leq \kappa(Y)\left\|\Delta C_{p_{l}}\right\|_{2},
$$

where $\Delta C_{p_{l}}=-e_{m}\left[\Delta q_{0}, \ldots, \Delta q_{m-1}\right]$ with $e_{m}=[0, \ldots, 0,1]^{T} \in \mathbb{R}^{m}$.

Proof. By applying Theorem 3.7 and the method of proof of Theorem 4.4, we get the desired result.

REMARK 4.6. Similar results can be obtained for the zeros of $p_{r}(z)$.

5. Numerical examples. In this section, we give some numerical examples to illustrate our results.

EXAMPLE 5.1. Let us consider a quaternionic matrix

$$
A=\left[\begin{array}{ccc}
2 \mathbf{i} & -2 \mathbf{j} & \mathbf{j}+\mathbf{k} \\
-\mathbf{k} & 2 & -1 \\
-\mathbf{j} & 1-\mathbf{i} & 1
\end{array}\right]
$$

The complex adjoint matrix of $A$ is

$$
\Psi_{A}=\left[\begin{array}{cc}
A_{1} & A_{2} \\
-\overline{A_{2}} & -\overline{A_{1}}
\end{array}\right], \quad A_{1}=\left[\begin{array}{ccc}
2 \mathbf{i} & 0 & 0 \\
0 & 2 & -1 \\
0 & 1-\mathbf{i} & 1
\end{array}\right], \quad A_{2}=\left[\begin{array}{ccc}
0 & -2 & 1+\mathbf{i} \\
-\mathbf{i} & 0 & 0 \\
-1 & 0 & 0
\end{array}\right] .
$$

Since $\Psi_{A}$ is diagonalizable, by Theorem $2.5, A$ is diagonalizable as well. The standard right eigenvalues of $A$ are $1,1+\mathbf{i}$, and $1+\mathbf{i}$, so $\Lambda_{s}(A)=\{1,1+\mathbf{i}\}$, where $1+\mathbf{i}$ appears with multiplicity 2. From [17, Theorem 3.1], there exist a nonsingular quaternionic matrix $Y$ such that $Y^{-1} A Y=\operatorname{diag}(1,1+\mathbf{i}, 1+\mathbf{i})$. Here,

$$
Y=\left[\begin{array}{ccc}
1+\mathbf{i} & \mathbf{i} & \mathbf{i} \\
\mathbf{j} & \mathbf{j} & 0 \\
-\mathbf{k} & -\mathbf{k} & -\mathbf{j}
\end{array}\right]
$$

Consider the perturbation matrix

$$
\Delta A=\left[\begin{array}{ccc}
0 & 0 & 0 \\
0 & 0 & \epsilon \\
\epsilon & \epsilon & \epsilon
\end{array}\right], \quad \epsilon=10^{-10}
$$

Then,

$$
A+\Delta A=\left[\begin{array}{ccc}
2 \mathbf{i} & -2 \mathbf{j} & \mathbf{j}+\mathbf{k} \\
-\mathbf{k} & 2 & -1+\epsilon \\
-\mathbf{j}+\epsilon & 1-\mathbf{i}+\epsilon & 1+\epsilon
\end{array}\right]
$$

Therefore, $\left.\|\Delta A\|_{2}=\epsilon \sqrt{(1}+\sqrt{2}\right)$ and $\Lambda_{s}(A+\Delta A)=\{1+1.0001 \mathbf{i}, 1.0001+0.9999 \mathbf{i}\}$, where $1+1.0001 \mathbf{i}$ appears with multiplicity 2 . The condition number of $Y$ is $\kappa(Y)=10.2193$ and

$$
\min _{\lambda_{i} \in \Lambda_{\mathbf{s}}(A)}\left\{\left|\lambda_{i}-\mu\right|\right\}=\min _{\eta_{i} \in \Lambda_{\mathbf{r}}(A), \xi \in \Lambda_{\mathbf{r}}(A+\Delta A)}\left\{\left|\eta_{i}-\xi\right|\right\}=0.0001
$$


Hence, Theorem 3.7 is verified.

EXAMPLE 5.2. Consider the quaternionic matrix

$$
A=\left[\begin{array}{cccc}
1 & -\mathbf{i} & -\mathbf{j} & \mathbf{k} \\
\mathbf{i} & 1 & -2 \mathbf{k} & \mathbf{j} \\
\mathbf{j} & 2 \mathbf{k} & 7 & -\mathbf{i} \\
-\mathbf{k} & -\mathbf{j} & \mathbf{i} & 1
\end{array}\right]
$$

The complex adjoint matrix of $A$ is

$$
\Psi_{A}=\left[\begin{array}{cc}
A_{1} & A_{2} \\
-\overline{A_{2}} & -\overline{A_{1}}
\end{array}\right], \quad A_{1}=\left[\begin{array}{cccc}
1 & -\mathbf{i} & 0 & 0 \\
\mathbf{i} & 1 & 0 & 0 \\
0 & 0 & 7 & -\mathbf{i} \\
0 & 0 & \mathbf{i} & 1
\end{array}\right], \quad A_{2}=\left[\begin{array}{cccc}
0 & 0 & -1 & \mathbf{i} \\
0 & 0 & -2 \mathbf{i} & 1 \\
1 & 2 \mathbf{i} & 0 & 0 \\
-\mathbf{i} & -1 & 0 & 0
\end{array}\right] .
$$

$A$ is Hermitian and, by Theorem $2.5, \Psi_{A}$ is Hermitian as well. The set of standard right eigenvalues of $A$ is $\Lambda_{s}(A)=\{-1,1,2,8\}$. Also, from Theorem 2.11, the matrix $A$ is unitarily diagonalizable, that is, $Y^{H} A Y=\operatorname{diag}(-1,1,2,8)$, where $Y \in \mathrm{M}_{n}(\mathbb{H})$ is a unitary matrix and $\Lambda_{s}(A)=\Lambda_{r}(A)$. Let

$$
\Delta A=\left[\begin{array}{llll}
0 & 0 & 0 & 0 \\
0 & 0 & 0 & \epsilon \\
0 & 0 & \epsilon & \epsilon \\
\epsilon & \epsilon & \epsilon & \epsilon
\end{array}\right], \quad \epsilon=10^{-3}, \quad A+\Delta A=\left[\begin{array}{cccc}
1 & -\mathbf{i} & -\mathbf{j} & \mathbf{k} \\
\mathbf{i} & 1 & -2 \mathbf{k} & \mathbf{j}+\epsilon \\
\mathbf{j} & 2 \mathbf{k} & 7+\epsilon & -\mathbf{i}+\epsilon \\
-\mathbf{k}+\epsilon & -\mathbf{j}+\epsilon & \mathbf{i}+\epsilon & 1+\epsilon
\end{array}\right]
$$

Then, $\|\Delta A\|_{2}=0.0024$ and

$$
\Lambda_{s}(A+\Delta A)=\{1.0003+0.0001 \mathbf{i}, 2.0005+0.0005 \mathbf{i},-0.9997+0.0003 \mathbf{i}, 8.0009\} .
$$

Since the matrix $A$ is Hermitian, $\kappa(Y)=1$ in Theorem 3.7. Moreover, $A$ is a central closed as well as a normal matrix, hence Corollary 3.8 is verified, too.

EXAMPLE 5.3. Let $t_{0}=2 \mathbf{i}=(0,2,0,0), t_{1}=\mathbf{j}=(0,0,1,0), t_{2}=\mathbf{k}=(0,0,0,1)$. Following [24], the quaternionic Vandermonde matrix is defined as

$$
A=\left[\begin{array}{ccc}
1 & 1 & 1 \\
t_{0} & t_{1} & t_{2} \\
t_{0}^{2} & t_{1}^{2} & t_{2}^{2}
\end{array}\right]=\left[\begin{array}{ccc}
1 & 1 & 1 \\
2 \mathbf{i} & \mathbf{j} & \mathbf{k} \\
-4 & -1 & -1
\end{array}\right]
$$

The complex adjoint matrix of $A$ is

$$
\Psi_{A}=\left[\begin{array}{cc}
A_{1} & A_{2} \\
-\overline{A_{2}} & -\overline{A_{1}}
\end{array}\right], \quad A_{1}=\left[\begin{array}{ccc}
1 & 1 & 1 \\
2 \mathbf{i} & 0 & 0 \\
-4 & -1 & 1
\end{array}\right], \quad A_{2}=\left[\begin{array}{ccc}
0 & 0 & 0 \\
0 & 1 & \mathbf{i} \\
0 & 0 & 0
\end{array}\right]
$$

The eigenvalues of $\Psi_{A}$ are

$$
\begin{array}{ccc}
0.8014+1.70007 \mathbf{i}, & 0.8014-1.70007 \mathbf{i}, & -0.4552+1.9952 \mathbf{i}, \\
-0.4552-1.9952 \mathbf{i}, & -0.3462+1.0469 \mathbf{i}, & -0.3462-1.0469 \mathbf{i},
\end{array}
$$

hence $\Psi_{A}$ is diagonalizable, and, by Theorem 2.5, $A$ is diagonalizable as well. The set of standard right eigenvalues is

$$
\Lambda_{s}(A)=\{0.8014+1.70007 \mathbf{i},-0.4552+1.9952 \mathbf{i},-0.3462+1.0469 \mathbf{i}\},
$$


and there exists an invertible matrix $Y \in M_{n}(\mathbb{H})$ such that $Y^{-1} A Y=\operatorname{diag}\left(\Lambda_{s}(A)\right)$. Let

$$
\Delta A=\left[\begin{array}{lll}
0 & 0 & 0 \\
0 & 0 & 0 \\
\epsilon & 0 & \epsilon
\end{array}\right], \quad \epsilon=10^{-3}, \quad A+\Delta A=\left[\begin{array}{ccc}
1 & 1 & 1 \\
2 \mathbf{i} & \mathbf{j} & \mathbf{k} \\
-4+\epsilon & -1 & -1+\epsilon
\end{array}\right] .
$$

Then, $\|\Delta A\|_{2}=1.4 \times 10^{-3}$ and

$$
\Lambda_{s}(A+\Delta A)=\{0.8016+1.7009 \mathbf{i},-0.4549+1.9950 \mathbf{i},-0.3457+1.0470 \mathbf{i}\} .
$$

Since $\kappa(Y) \geq 1$, we have $\kappa(Y)\|\Delta A\|_{2} \geq 0.0014$. Also,

$$
\min _{\lambda_{i} \in \Lambda_{\mathbf{s}}(A)}\left\{\left|\lambda_{i}-\mu\right|\right\}=\min _{\substack{\eta_{i} \in \Lambda_{\mathbf{r}}(A) \\ \xi \in \Lambda_{\mathbf{r}}(A+\Delta A)}}\left\{\left|\eta_{i}-\xi\right|\right\}=0.0002
$$

hence Theorem 3.7 is verified.

Acknowledgement. We thank the associate editor Professor Dario A. Bini and the anonymous referee for their useful suggestions for the improvement of this article.

\section{REFERENCES}

[1] S. L. AdLER, Quaternionic Quantum Mechanics and Quantum Fields, Oxford University Press, New York, 1995.

[2] S. S. AHMAD AND I. ALI, Bounds for eigenvalues of matrix polynomials over quaternion division algebra, Adv. Appl. Clifford Algebr., 26 (2016), pp. 1095-1125.

[3] L Localization theorems for matrices and bounds for the zeros of polynomials over a quaternion division algebra, Filomat, 32 (2018), pp. 553-573.

[4] F. L. BAUER AND C. T. FIKE, Norms and exclusion theorems, Numer. Math., 2 (1960), pp. 137-141.

[5] E. K. CHU, Generalization of the Bauer-Fike theorem, Numer. Math., 49 (1986), pp. 685-691.

[6] - Perturbarion of eigenvalues for matrix polynomials via the Bauer-Fike theorems, SIAM J. Matrix Anal. Appl., 25 (2003), pp. 551-573.

[7] S. DE Leo, G. DUCATI, AND V. LeONARDI, Zeros of unilateral quaternionic polynomials, Electron. J. Linear Algebra, 15 (2006), pp. 297-313.

[8] S. C. EISENSTAT AND I. C. F. IPSEN, Three absolute perturbation bounds for matrix eigenvalues imply relative bounds, SIAM J. Matrix Anal. Appl., 20 (1998), pp. 149-158.

[9] D. R. FARENICK AND B. A. F. PIDKowich, The spectral theorem in quaternions, Linear Algebra Appl., 371 (2003), pp. 75-102.

[10] A. Galántai And C. J. Hegedüs, Perturbation bounds for polynomials, Numer. Math., 109 (2008), pp. $77-100$

[11] G. H. Golub and C. F. Van Loan, Matrix Computations, 3rd ed., Johns Hopkins University Press, Baltimore, 1996

[12] B. Gordon AND T. S. MotZKin, On the zeros of polynomials over division rings, Trans. Amer. Math. Soc., 116 (1965), pp. 218-226.

[13] R. A. Horn and C. R. Johnson, Matrix Analysis, Cambridge University Press, Cambridge, 1994.

[14] I. C. F. IPSEN, A note on unifying absolute and relative perturbation bounds, Linear Algebra Appl., 358 (2003), pp. 239-253.

[15] D. JANOVSKÁ AND G. OPFER, The classification and the computation of the zeros of quaternionic, two-sided polynomials, Numer. Math., 115 (2010), pp. 81-100.

[16] - A note on the computation of all zeros of simple quaternionic polynomials, SIAM J. Numer. Anal., 48 (2010), pp. 244-256.

[17] T. JIANG, Algebraic methods for diagonalization of a quaternion matrix in quaternionic quantum theory, J. Math. Phys., 46 (2005), Art. Id. 052106, 8 pages.

[18] B. KALANTARI, Algorithms for quaternion polynomial root-finding, J. Complexity, 29 (2013), pp. 302-322.

[19] J. B. KUIPERS, Quaternions and Rotation Sequences, Princeton University Press, Princeton, 1999.

[20] T. A. LORING, Factorization of matrices of quaternions, Expo. Math., 30 (2012), pp. 250-267.

[21] V. Mehrmann And H. Xu, An analysis of the pole placement problem. I. The single-input case, Electron. Trans. Numer. Anal., 4 (1996), pp. 89-105.

http://etna.ricam.oeaw.ac.at/vol.4.1996/pp89-105.dir/pp89-105.pdf 
[22] — An analysis of the pole placement problem. II. The multi-input case, Electron. Trans. Numer. Anal., 5 (1997), pp. 77-97.

http://etna.ricam.oeaw.ac.at/vol.5.1997/pp77-97.dir/pp77-97.pdf

[23] I. Niven, Equations in quaternions, Amer. Math. Monthly, 48 (1941), pp. 654-661.

[24] G. OPfER, Polynomials and Vandermonde matrices over the field of quaternions, Electron. Trans. Numer. Anal., 36 (2009), pp. 9-16.

http://etna.ricam.oeaw.ac.at/vol.36.2009-2010/pp9-16.dir/pp9-16.pdf

[25] R. PereIRA, Quaternionic Polynomials and Behavioral Systems, PhD. Thesis, Departement of Math., University of Aveiro, Aveiro, 2006.

[26] A. Pogorui AND M. SHAPIRO, On the structure of the set of zeros of quaternionic polynomials, Complex Var. Elliptic Equ., 40 (2004), pp. 379-389.

[27] L. Rodman, Canonical forms for mixed symmetric-skewsymmetric quaternion matrix pencils, Linear Algebra Appl., 424 (2007), pp. 184-221.

[28] - Topics in Quaternion Linear Algebra, Princeton University Press, Princeton, 2014.

[29] R. Serôdio, E. Pereira, AND J. VitóRIA, Computing the zeros of quaternion polynomials, Comput. Math. Appl., 42 (2001), pp. 1229-1237.

[30] X. SHI AND Y. WEI, A sharp version of Bauer-Fike's theorem, J. Comput. Appl. Math., 236 (2012), pp. $3218-$ 3227.

[31] N. A. Wiegmann, Some theorems on matrices with real quaternion entries, Canadian J. Math., 7 (1955), pp. 191-201.

[32] L. A. WolF, Similarity of matrices in which the elements are real quaternions, Bull. Amer. Math. Soc., 42 (1936), pp. 737-743.

[33] F. Zhang, Quaternions and matrices of quaternions, Linear Algebra Appl., 251 (1997), pp. 21-57. 